\title{
Adaptive Observer-Based Fault-Tolerant Control Design for Uncertain Systems
}

\author{
Huaming Qian, ${ }^{1}$ Yu Peng, ${ }^{1}$ and Mei Cui ${ }^{2}$ \\ ${ }^{1}$ College of Automation, Harbin Engineering University, Harbin 150001, China \\ ${ }^{2}$ China Ship Development \& Design Center, Wuhan 430064, China \\ Correspondence should be addressed to Yu Peng; lyricday@sina.cn
}

Received 15 October 2014; Revised 10 January 2015; Accepted 12 January 2015

Academic Editor: Xinggang Yan

Copyright (c) 2015 Huaming Qian et al. This is an open access article distributed under the Creative Commons Attribution License, which permits unrestricted use, distribution, and reproduction in any medium, provided the original work is properly cited.

\begin{abstract}
This study focuses on the design of the robust fault-tolerant control (FTC) system based on adaptive observer for uncertain linear time invariant (LTI) systems. In order to improve robustness, rapidity, and accuracy of traditional fault estimation algorithm, an adaptive fault estimation algorithm (AFEA) using an augmented observer is presented. By utilizing a new fault estimator model, an improved AFEA based on linear matrix inequality (LMI) technique is proposed to increase the performance. Furthermore, an observer-based state feedback fault-tolerant control strategy is designed, which guarantees the stability and performance of the faulty system. Moreover, the adaptive observer and the fault-tolerant controller are designed separately, whose performance can be considered, respectively. Finally, simulation results of an aircraft application are presented to illustrate the effectiveness of the proposed design methods.
\end{abstract}

\section{Introduction}

With the rapid development of system complexity and integration, the possibility of system failures including actuator, sensor, and process faults raises increasingly, which may drastically change the system behavior and result in performance degradation or even instability. Therefore, safety and reliability have become important indicators to measure the system performance. Fault-tolerant control (FTC) is viewed as one of the most effective methods to increase system safety and reliability and has become an attractive topic, which has received considerable attention in the field of the industrial control and engineering research during the last couple of decades [1-3]. Depending on how redundancies are being utilized, current FTC can be classified into two categories, namely, passive FTC [4-8] and active FTC [917]. Passive FTC uses control laws to make the system robust with respect to possible faults, which are considered as a special kind of uncertainties. In contrast, active FTC depends on real-time fault information, which is provided by fault estimation module. Based on the obtained fault information, an additional control law can be designed to preserve the stability of the overall system and maintain an acceptable level of performance in the presence of faults.

From the above design procedure of active FTC, it can be seen that fault estimation is a key step, of which many effective methods have been developed in the last decade, such as sliding mode observer approach [18-20], proportional integral observer method [21,22], adaptive observer technique [23-31], and intelligence algorithm based on neural network [32-34]. Among these methods, many researchers have paid attention to fault estimation based on adaptive observer. However, the main difficulties in the use of adaptive observer focus on the following aspects. First, the existence conditions of adaptive observer are not given clearly, which adds difficulties to verify whether adaptive observer exists for the monitored system [24]. Second, adaptive observer cannot deal with uncertain systems containing unknown disturbances very well $[25,27]$. Thirdly, since active FTC is based on fault estimation, both time delay and inaccuracy of fault estimation can have detrimental impacts on the closedloop system performance. But conventional adaptive fault estimation observer can only apply to systems with constant faults. It leads to a poor performance when time-varying 
faults exist [23]. Therefore, the performance requirements of fault estimation, that is, rapidity and accuracy, play an important role in the design of adaptive observer.

On the basis of the obtained on-line fault information, a fault-tolerant controller can be designed. In general cases, the state variables of practical systems are unavailable. So an observer-based state feedback fault-tolerant controller can handle this situation $[26,28,30]$. However, the coupling problem between the adaptive fault estimation observer and the fault-tolerant controller increases the design difficulty apparently.

The aforementioned difficulties are the motivations of this study. Therefore, the aim of this paper is to analyze and develop an improved framework of robust FTC based on adaptive observer for uncertain linear time invariant (LTI) systems. The main contributions are as follows. First, by using a normal fault estimator model and constructing an augmented observer, an adaptive fault estimation algorithm (AFEA) utilizes the $H_{\infty}$ theory to inhibit the influence of disturbances on system performance. Second, by presenting a new fault estimator model, an improved AFEA is proposed, which designs the observer gain matrices based on linear matrix inequality (LMI) constraint technique and the generalized inverse matrix theory, to improve the rapidity and accuracy of fault estimation. Moreover, the improved AFEA introduces the convergence set and the convergence rate to choose the uncertain parameter in the fault estimator model. Thirdly, based on the improved AFEA, an observerbased state feedback fault-tolerant controller (OSFFTC) is constructed separately, which considers the performance of the adaptive observer and the fault-tolerant controller, respectively. A detailed proof is given to demonstrate that the proposed fault-tolerant controller can maintain the stability and performance of the faulty system. Furthermore, this paper introduces the existence conditions of two kinds of adaptive observers and the fault-tolerant controller in detail.

This paper is organized as follows. The system description is presented in Section 2. Sections 3 and 4, respectively, introduce AFEA design and improved AFEA design. Section 5 gives the design algorithm of the fault-tolerant controller based on improved AFEA. Simulation results of an aircraft plant are given in Section 6 to illustrate the effectiveness of the proposed design techniques, followed by some concluding remarks in Section 7.

\section{System Description}

Consider the following LTI system with faults and unknown disturbances:

$$
\begin{gathered}
\dot{\mathbf{x}}(t)=\mathbf{A} \mathbf{x}(t)+\mathbf{B u}(t)+\mathbf{E f}(t)+\mathbf{D} \boldsymbol{w}(t), \\
\mathbf{y}(t)=\mathbf{C} \mathbf{x}(t),
\end{gathered}
$$

where $\mathbf{x}(t) \in \mathbf{R}^{n}$ is the state vector, $\mathbf{u}(t) \in \mathbf{R}^{m}$ is the input vector, $\mathbf{y}(t) \in \mathbf{R}^{p}$ is the output vector, $\mathbf{f}(t) \in \mathbf{R}^{r}$ represents the process and actuator fault, and $\boldsymbol{\omega}(t) \in \mathbf{R}^{d}$ is the unknown disturbance including external interference and model uncertainties (i.e., $\Delta \mathbf{A x}(t)+\Delta \mathbf{B u}(t))$ [35], which is supposed to be bounded; that is, $\|\boldsymbol{\omega}(t)\|_{2} \leq \omega_{0}$, where $\|\cdot\|_{2}$ denotes 2-norm and $0 \leq \omega_{0}<\infty$. A, B, C, D, and $\mathbf{E}$ are constant real matrices of appropriate dimensions, the matrix $\mathbf{B}$ is of full column rank, and the matrix $\mathbf{C}$ is of full row rank. The pairs $(\mathbf{A}, \mathbf{B})$ and $(\mathbf{A}, \mathbf{C})$ are supposed to be, respectively, controllable and observable.

Faults are assumed to be zero prior to the failure time and nonzero and time-varying after the fault occurrence. It is assumed that $\mathbf{f}(t)$ and its time derivative $\dot{\mathbf{f}}(t)$ are bounded; that is, there exist two constants $f_{0}$ and $f_{1}$ such that $\|\mathbf{f}(t)\|_{2} \leq$ $f_{0}$ and $\|\dot{\mathbf{f}}(t)\|_{2} \leq f_{1}$.

In order to detect and estimate the faults, the fault estimation observer is constructed as follows:

$$
\begin{aligned}
\dot{\hat{\mathbf{x}}}(t)=\mathbf{A} \widehat{\mathbf{x}}(t)+\mathbf{B u}(t)+\mathbf{E} \widehat{\mathbf{f}}(t)+\mathbf{L}(\mathbf{y}(t)-\widehat{\mathbf{y}}(t)), \\
\widehat{\mathbf{y}}(t)=\mathbf{C} \widehat{\mathbf{x}}(t),
\end{aligned}
$$

where $\widehat{\mathbf{x}}(t) \in \mathbf{R}^{n}$ is the observer state vector, that is, the state estimation vector, $\widehat{\mathbf{y}}(t) \in \mathbf{R}^{p}$ is the observer output vector, $\widehat{\mathbf{f}}(t) \in \mathbf{R}^{r}$ is the fault estimation vector, and $\mathbf{L} \in \mathbf{R}^{n \times p}$ is the observer gain matrix required to design.

Denote

$$
\begin{gathered}
\mathbf{e}_{x}(t)=\mathbf{x}(t)-\widehat{\mathbf{x}}(t), \quad \mathbf{e}_{f}(t)=\mathbf{f}(t)-\widehat{\mathbf{f}}(t), \\
\mathbf{e}_{y}(t)=\mathbf{y}(t)-\widehat{\mathbf{y}}(t) .
\end{gathered}
$$

Then the error dynamics are described by

$$
\begin{gathered}
\dot{\mathbf{e}}_{x}(t)=(\mathbf{A}-\mathbf{L C}) \mathbf{e}_{x}(t)+\mathbf{E} \mathbf{e}_{f}(t)+\mathbf{D} \boldsymbol{w}(t), \\
\mathbf{e}_{y}(t)=\mathbf{C} \mathbf{e}_{x}(t),
\end{gathered}
$$

where $\mathbf{e}_{x}(t) \in \mathbf{R}^{n}$ is the state estimation error vector, $\mathbf{e}_{y}(t) \epsilon$ $\mathbf{R}^{p}$ is the output estimation error vector, and $\mathbf{e}_{f}(t) \in \mathbf{R}^{r}$ is the fault estimation error vector.

In this paper, two fault estimation algorithms, that is, an AFEA and an improved AFEA, will be presented. The AFEA constructs an augmented observer with multiobjective constraint to inhibit the influence of disturbances on fault estimation performance. Then, the improved AFEA uses a new fault estimator model, which plays a major role in improving the rapidity and accuracy of fault estimation. An LMI-based method and the introduction of the convergence rate are used to calculate the observer gain matrices and the unknown parameter. Furthermore, two kinds of fault estimation observers can achieve the estimation of both states and faults simultaneously.

On the basis of the obtained on-line state and fault information, an OSFFTC is designed to stabilize the closedloop system in the presence of faults. Figure 1 illustrates the structure of the proposed FTC system based on adaptive observer.

\section{AFEA Design}

In this section, AFEA will be introduced. First, one lemma is given before the design of adaptive fault estimation observer (AFEO). 


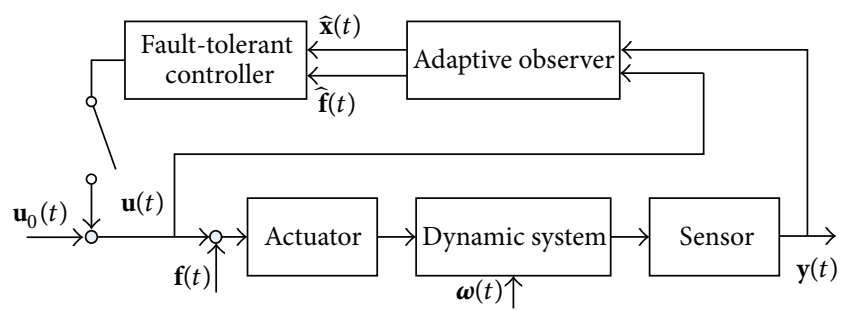

FIGURE 1: The structure of the proposed FTC system based on adaptive observer.

Lemma 1 (see [37]). The eigenvalues of a given matrix $\mathbf{A} \in$ $\mathbf{R}^{n \times n}$ belong to the closed circular region $\mathrm{D}(\lambda, \tau)$ with center $\lambda+j_{0}$ and radius $\tau$ if and only if there exists a symmetric positive definite matrix $\mathrm{P} \in \mathbf{R}^{n \times n}$ such that the following condition holds:

$$
\left[\begin{array}{cc}
-\mathbf{P} & \mathbf{P}\left(\mathbf{A}-\lambda \mathbf{I}_{n}\right) \\
* & -\tau^{2} \mathbf{P}
\end{array}\right] \leq 0,
$$

where, here and everywhere in the sequel, * denotes the symmetric elements in a symmetric matrix and $\mathbf{I}_{n}$ denotes the identity matrix with dimension $n \times n$ (this dimension definition will also be used in the sequel).

Considering the normal adaptive fault estimator model

$$
\dot{\overrightarrow{\mathbf{f}}}(t)=\boldsymbol{\Gamma}\left(\widehat{\mathbf{f}}(t)+\mathbf{R e}_{y}(t)\right),
$$

where $\mathbf{R} \in \mathbf{R}^{r \times p}$ is the gain matrix needed to design and the symmetric positive definite matrix $\Gamma \in \mathbf{R}^{r \times r}$ is the adaptive learning rate.

The adaptive learning rate $\boldsymbol{\Gamma}$ is an independent performance index function, which means different adaptive learning rate may have different impact on the fault estimation performance; particularly it may amplify the influence of disturbances [25]. Meanwhile, there is no unified standard for the selection of the adaptive learning rate $\Gamma$. Therefore, considering the fact that how to choose the adaptive learning rate $\Gamma$ is not the research emphasis in this paper, it is assumed that $\boldsymbol{\Gamma}=\mathbf{I}_{r}$ in this paper (including the design of improved AFEA). Then (8) can be written as

$$
\dot{\hat{\mathbf{f}}}(t)=\widehat{\mathbf{f}}(t)+\operatorname{Re}_{y}(t) .
$$

Considering time-varying faults, the time derivative of $\mathbf{e}_{f}(t)$ is

$$
\begin{aligned}
\dot{\mathbf{e}}_{f}(t) & =\dot{\mathbf{f}}(t)-\dot{\widehat{\mathbf{f}}}(t)=\dot{\mathbf{f}}(t)-\widehat{\mathbf{f}}(t)-\mathbf{R e}_{y}(t) \\
& =-\mathbf{R C e}_{x}(t)+\mathbf{f}(t)-\widehat{\mathbf{f}}(t)-\mathbf{f}(t)+\dot{\mathbf{f}}(t) \\
& =-\mathbf{R C e}_{x}(t)+\mathbf{e}_{f}(t)-\mathbf{f}(t)+\dot{\mathbf{f}}(t) .
\end{aligned}
$$

Use the error systems (5) and (10) to construct an augmented system

$$
\dot{\widetilde{\mathbf{e}}}(t)=(\widetilde{\mathbf{A}}-\widetilde{\mathbf{L}} \widetilde{\mathbf{C}}) \widetilde{\mathbf{e}}(t)+\widetilde{\mathbf{D}} \widetilde{\boldsymbol{w}}(t),
$$

where

$$
\begin{gathered}
\widetilde{\mathbf{e}}(t)=\left[\begin{array}{l}
\mathbf{e}_{x}(t) \\
\mathbf{e}_{f}(t)
\end{array}\right], \quad \widetilde{\boldsymbol{\omega}}(t)=\left[\begin{array}{c}
\boldsymbol{\omega}(t) \\
\mathbf{f}(t) \\
\dot{\mathbf{f}}(t)
\end{array}\right], \quad \widetilde{\mathbf{A}}=\left[\begin{array}{cc}
\mathbf{A} & \mathbf{E} \\
0 & \mathbf{I}_{r}
\end{array}\right], \\
\widetilde{\mathbf{L}}=\left[\begin{array}{l}
\mathbf{L} \\
\mathbf{R}
\end{array}\right], \quad \widetilde{\mathbf{C}}=\left[\begin{array}{ll}
\mathbf{C} & 0
\end{array}\right], \quad \widetilde{\mathbf{D}}=\left[\begin{array}{ccc}
\mathbf{D} & 0 & 0 \\
0 & -\mathbf{I}_{r} & \mathbf{I}_{r}
\end{array}\right] .
\end{gathered}
$$

Remark 2. Not considering the disturbance term $\widetilde{\boldsymbol{\omega}}(t)$ and according to the asymptotically equivalent condition of full dimension state observer [36], it can be obtained that the necessary and sufficient condition for the existence of AFEO is that the pair $(\widetilde{\mathbf{A}}, \widetilde{\mathbf{C}})$ is completely observable, which is equivalent to the fact that there exist a symmetric positive definite matrix $\widetilde{\mathbf{P}} \in \mathbf{R}^{(n+r) \times(n+r)}$ and the matrix $\widetilde{\mathbf{L}} \in \mathbf{R}^{(n+r) \times p}$ such that the following inequality holds:

$$
\widetilde{\mathbf{P}}(\widetilde{\mathbf{A}}-\widetilde{\mathbf{L}} \widetilde{\mathbf{C}})+(\widetilde{\mathbf{A}}-\widetilde{\mathbf{L}} \widetilde{\mathbf{C}})^{T} \widetilde{\mathbf{P}}<0
$$

From (11), it is easy to see that two unknown observer gain matrices $\mathbf{L}$ and $\mathbf{R}$ are put into one augmented matrix $\widetilde{\mathbf{L}}$. So the design of AFEO can be expressed as calculating the optimal observer gain matrix $\widetilde{\mathbf{L}}$ to stabilize the error system (11) and minimize $\widetilde{\mathbf{e}}(t)$ as much as possible.

Theorem 3. Given a circular region $\mathrm{D}\left(\lambda_{1}, \tau_{1}\right)$ and a prescribed $H_{\infty}$ performance level $\gamma_{1}>0$, if there exist a symmetric positive definite matrix $\widetilde{\mathbf{P}} \in \mathbf{R}^{(n+r) \times(n+r)}$ and a matrix $\widetilde{\mathbf{Y}} \in \mathbf{R}^{(n+r) \times p}$ such that the following conditions hold

$$
\begin{gathered}
{\left[\begin{array}{cc}
-\widetilde{\mathbf{P}} & \widetilde{\mathbf{P}} \widetilde{\mathbf{A}}-\widetilde{\mathbf{Y}} \widetilde{\mathbf{C}}-\lambda_{1} \widetilde{\mathbf{P}} \\
* & -\tau_{1}^{2} \widetilde{\mathbf{P}}
\end{array}\right]<0,} \\
{\left[\begin{array}{ccc}
\psi_{11} & \widetilde{\mathbf{P}} \widetilde{\mathbf{D}} & \mathbf{I}_{n+r} \\
* & -\gamma_{1} \mathbf{I}_{d+2 r} & 0 \\
* & * & -\gamma_{1} \mathbf{I}_{n+r}
\end{array}\right]<0,}
\end{gathered}
$$

where $\psi_{11}=\widetilde{\mathbf{P}} \widetilde{\mathbf{A}}+\widetilde{\mathbf{A}}^{T} \widetilde{\mathbf{P}}-\widetilde{\mathbf{Y}} \widetilde{\mathbf{C}}-\widetilde{\mathbf{C}}^{T} \widetilde{\mathbf{Y}}^{T}$ and $\widetilde{\mathbf{Y}}=\widetilde{\mathbf{P}} \widetilde{\mathbf{L}}$, then the eigenvalues of $(\widetilde{\mathbf{A}}-\widetilde{\mathbf{L}} \widetilde{\mathbf{C}})$ belong to $\mathrm{D}\left(\lambda_{1}, \tau_{1}\right)$, the error dynamics (11) satisfy the $H_{\infty}$ performance index $\|\widetilde{\mathbf{e}}(t)\|_{2}<\gamma_{1}\|\widetilde{\boldsymbol{\omega}}(t)\|_{2}$, and the AFEO gain matrix can be obtained by $\widetilde{\mathbf{L}}=\widetilde{\mathbf{P}}^{-1} \widetilde{\mathbf{Y}}$.

Proof. Condition (14): according to Lemma 1, setting ( $\widetilde{\mathbf{A}}-$ $\widetilde{\mathbf{L}} \widetilde{\mathbf{C}}) \rightarrow \mathbf{A}$ and $\widetilde{\mathbf{P}} \rightarrow \mathrm{P}$, it is easy to prove that if condition (14) is satisfied, the eigenvalues of $(\widetilde{\mathbf{A}}-\widetilde{\mathbf{L}} \widetilde{\mathbf{C}})$ belong to $D\left(\lambda_{1}, \tau_{1}\right)$.

Condition (15): consider the following Lyapunov function:

$$
V(t)=\widetilde{\mathbf{e}}^{T}(t) \widetilde{\mathbf{P}} \widetilde{\mathbf{e}}(t) .
$$


According to (11), its time derivative is

$$
\begin{aligned}
\dot{V}(t)= & \dot{\tilde{\mathbf{e}}}^{T}(t) \widetilde{\mathbf{P}} \widetilde{\mathbf{e}}(t)+\widetilde{\mathbf{e}}^{T}(t) \widetilde{\mathbf{P}} \dot{\tilde{\mathbf{e}}}(t) \\
= & \widetilde{\mathbf{e}}^{T}(t)\left(\widetilde{\mathbf{P}}(\widetilde{\mathbf{A}}-\widetilde{\mathbf{L}} \widetilde{\mathbf{C}})+(\widetilde{\mathbf{A}}-\widetilde{\mathbf{L}} \widetilde{\mathbf{C}})^{T} \widetilde{\mathbf{P}}\right) \widetilde{\mathbf{e}}(t) \\
& +\widetilde{\mathbf{e}}^{T}(t) \widetilde{\mathbf{P}} \widetilde{\mathbf{D}} \widetilde{\boldsymbol{\omega}}(t)+\widetilde{\boldsymbol{\omega}}^{T}(t) \widetilde{\mathbf{D}}^{T} \widetilde{\mathbf{P}} \widetilde{\mathbf{e}}(t) .
\end{aligned}
$$

Define

$$
J_{1}=\int_{0}^{T}\left[\frac{1}{\gamma_{1}} \widetilde{\mathbf{e}}^{T}(t) \widetilde{\mathbf{e}}(t)-\gamma_{1} \widetilde{\boldsymbol{\omega}}^{T}(t) \widetilde{\boldsymbol{\omega}}(t)\right] d t .
$$

Under the zero initial condition, that is, $V(0)=0$, it follows that

$$
\begin{aligned}
J_{1}= & \int_{0}^{T}\left[\frac{1}{\gamma_{1}} \widetilde{\mathbf{e}}^{T}(t) \widetilde{\mathbf{e}}(t)-\gamma_{1} \widetilde{\boldsymbol{\omega}}^{T}(t) \widetilde{\boldsymbol{\omega}}(t)+\dot{V}(t)\right] d t \\
& -(V(t)-V(0)) \\
\leq & \int_{0}^{T}\left[\frac{1}{\gamma_{1}} \widetilde{\mathbf{e}}^{T}(t) \widetilde{\mathbf{e}}(t)-\gamma_{1} \widetilde{\boldsymbol{\omega}}^{T}(t) \widetilde{\boldsymbol{\omega}}(t)+\dot{V}(t)\right] d t .
\end{aligned}
$$

Substituting (17) into (19) yields

$$
J_{1} \leq \int_{0}^{T}\left[\begin{array}{lll}
\widetilde{\mathbf{e}}^{T}(t) & \widetilde{\boldsymbol{\omega}}^{T}(t)
\end{array}\right] \boldsymbol{\Omega}\left[\begin{array}{c}
\widetilde{\mathbf{e}}(t) \\
\widetilde{\boldsymbol{\omega}}(t)
\end{array}\right] d t
$$

where

$$
\boldsymbol{\Omega}=\left[\begin{array}{ccc}
\frac{1}{\gamma_{1}} \mathbf{I}_{n+r}+\psi_{11} & \widetilde{\mathbf{P}} \widetilde{\mathbf{D}} \\
* & -\gamma_{1} \mathbf{I}_{d+2 r}
\end{array}\right]
$$

From above, it can be obtained that if LMI (15) holds, $\Omega<0$ holds with the application of Schur complement lemma. Further, one can obtain $J_{1}<0$; that is, $\|\widetilde{\mathbf{e}}(t)\|_{2}<$ $\gamma_{1}\|\widetilde{\boldsymbol{\omega}}(t)\|_{2}$. Meanwhile, condition (14) guarantees the observer system asymptotic stability according to Lyapunov stability theory [36]; that is, existence condition (13) of AFEO holds. Therefore, if conditions (14) and (15) hold, the error dynamics (11) are stable and satisfy the $H_{\infty}$ performance index $\|\widetilde{\mathbf{e}}(t)\|_{2}<$ $\gamma_{1}\|\widetilde{\boldsymbol{\omega}}(t)\|_{2}$.

Remark 4. In Theorem 3, an AFEO design algorithm based on multiobjective constraint is proposed, where the purpose of introducing pole-clustering in the special region of left-half plane (14) is to control the state and fault estimation transient performance [37], and simultaneously, LMI constraint (15) is used to improve the accuracy of state and fault estimation by inhibiting the influence of disturbances on $\widetilde{\mathbf{e}}(t)$.

\section{Improved AFEA Design}

In this section, the main ideas of improved AFEA will be presented.
Define a new fault estimator model

$$
\dot{\widehat{\mathbf{f}}}(t)=\mathbf{R}_{1} \widehat{\mathbf{f}}(t)+\mathbf{R}_{2}\left(\dot{\mathbf{e}}_{y}(t)+\sigma \mathbf{e}_{y}(t)\right),
$$

where $\mathbf{R}_{1} \in \mathbf{R}^{r \times r}$ and $\mathbf{R}_{2} \in \mathbf{R}^{r \times p}$ are the gain matrices needed to design and $\sigma$ is a positive scalar factor. The way to choose constant $\sigma$ will be given later.

Remark 5. Compared with the fault estimator model in AFEA, the introduction of the proportional gain $\mathbf{R}_{1}$ rather than the identity matrix provides some degree of freedom. And the differential item $\dot{\mathbf{e}}_{y}(t)$ can reduce the transition process of fault estimation, which plays an important role to improve the rapidity and accuracy of fault estimation.

Before introducing the design method, one assumption and one lemma are given.

Assumption 6. There exist a symmetric positive definite matrix $\mathbf{P} \in \mathbf{R}^{n \times n}$, a matrix $\mathbf{L} \in \mathbf{R}^{n \times p}$, and a matrix $\mathbf{R}_{2} \in \mathbf{R}^{r \times p}$ to satisfy the following conditions:

$$
\begin{gathered}
\mathbf{P}(\mathbf{A}-\mathbf{L} \mathbf{C})+(\mathbf{A}-\mathbf{L C})^{T} \mathbf{P}<0, \\
\mathbf{E}^{T} \mathbf{P}=\mathbf{R}_{2} \mathbf{C} .
\end{gathered}
$$

Remark 7. As same as Remark 2, condition (23) is equivalent to the fact that the pair $(\mathbf{A}, \mathbf{C})$ is observable. Condition (24) is a requirement of the algorithm, which will be used in the design of improved adaptive fault estimation observer (IAFEO). Therefore, under assumption (24), the observability of the pair $(\mathbf{A}, \mathbf{C})$ can be regarded as the judgment of whether IAFEO exists [38].

Lemma 8 (see [27]). For any scalar $\mu>0$ and real vectors a and $\mathbf{b}$, the following inequality holds:

$$
2 \mathbf{a}^{T} \mathbf{b} \leq \frac{1}{\mu} \mathbf{a}^{T} \mathbf{a}+\mu \mathbf{b}^{T} \mathbf{b} .
$$

Considering time-varying faults, the time derivative of $\mathbf{e}_{f}(t)$ can be obtained as

$$
\begin{aligned}
\dot{\mathbf{e}}_{f}(t)= & \dot{\mathbf{f}}(t)-\dot{\hat{\mathbf{f}}}(t)=\dot{\mathbf{f}}(t)-\mathbf{R}_{1} \widehat{\mathbf{f}}(t)-\mathbf{R}_{2}\left(\dot{\mathbf{e}}_{y}(t)+\sigma \mathbf{e}_{y}(t)\right) \\
= & \mathbf{R}_{1} \mathbf{f}(t)-\mathbf{R}_{1} \widehat{\mathbf{f}}(t)-\mathbf{R}_{2}\left(\dot{\mathbf{e}}_{y}(t)+\sigma \mathbf{e}_{y}(t)\right) \\
& -\mathbf{R}_{1} \mathbf{f}(t)+\dot{\mathbf{f}}(t) \\
= & \mathbf{R}_{1} \mathbf{e}_{f}(t)-\mathbf{R}_{2}\left(\dot{\mathbf{e}}_{y}(t)+\sigma \mathbf{e}_{y}(t)\right)-\mathbf{R}_{1} \mathbf{f}(t)+\dot{\mathbf{f}}(t) .
\end{aligned}
$$

According to (5) and (26), except for the matrices $\mathbf{L}, \mathbf{R}_{1}$, and $\mathbf{R}_{2}$, other parameter matrices are known. Therefore, the design of IAFEO can be described as calculating the optimal observer gain matrices $\mathbf{L}, \mathbf{R}_{1}$, and $\mathbf{R}_{2}$ to stabilize the error systems (5) and (26) and minimize $\mathbf{e}_{x}(t)$ and $\mathbf{e}_{f}(t)$ as much as possible.

Theorem 9. Given a circular region $\mathrm{D}\left(\lambda_{2}, \tau_{2}\right)$, a prescribed $H_{\infty}$ performance level $\gamma_{2}>0$, and a scalar $\sigma>0$, if there exist a symmetric positive definite matrix $\mathbf{P} \in \mathbf{R}^{n \times n}$, a symmetric matrix $\mathbf{R}_{1} \in \mathbf{R}^{r \times r}$, and a matrix $\mathbf{Y} \in \mathbf{R}^{n \times p}$ such that the following conditions hold 


$$
\begin{gathered}
{\left[\begin{array}{rrrr}
-\mathbf{P} & \mathbf{P A}-\mathbf{Y C}-\lambda_{2} \mathbf{P} \\
* & -\tau_{2}^{2} \mathbf{P}
\end{array}\right]<0,} \\
{\left[\begin{array}{ccccccc}
\boldsymbol{\varphi}_{11} & -\frac{1}{\sigma}\left(\mathbf{A}^{T} \mathbf{P E}-\mathbf{C}^{T} \mathbf{Y}^{T} \mathbf{E}\right) & \mathbf{P D} & 0 & 0 & \mathbf{I}_{n} & 0 \\
* & -2 \frac{1}{\sigma} \mathbf{E}^{T} \mathbf{P E}+2 \frac{1}{\sigma} \mathbf{R}_{1} & -\frac{1}{\sigma} \mathbf{E}^{T} \mathbf{P D} & -\frac{1}{\sigma} \mathbf{R}_{1} & \frac{1}{\sigma} \mathbf{I}_{r} & 0 & \mathbf{I}_{r} \\
* & * & -\gamma_{2} \mathbf{I}_{d} & 0 & 0 & 0 & 0 \\
* & * & * & -\gamma_{2} \mathbf{I}_{r} & 0 & 0 & 0 \\
* & * & * & * & -\gamma_{2} \mathbf{I}_{r} & 0 & 0 \\
* & * & * & * & * & -\gamma_{2} \mathbf{I}_{n} & 0 \\
* & * & * & * & * & * & -\gamma_{2} \mathbf{I}_{r}
\end{array}\right]<0,}
\end{gathered}
$$

where $\boldsymbol{\varphi}_{11}=\mathbf{P A}+\mathbf{A}^{T} \mathbf{P}-\mathbf{Y C}-\mathbf{C}^{T} \mathbf{Y}^{T}$ and $\mathbf{Y}=\mathbf{P L}$, then the eigenvalues of $(\mathbf{A}-\mathbf{L} \mathbf{C})$ belong to $\mathrm{D}\left(\lambda_{2}, \tau_{2}\right)$, the error dynamics (5) and (26) satisfy the $H_{\infty}$ performance index $\|\widetilde{\mathbf{e}}(t)\|_{2}<$ $\gamma_{2}\|\widetilde{\boldsymbol{\omega}}(t)\|_{2}$, and the IAFEO gain matrices can be obtained by $\mathbf{L}=$ $\mathbf{P}^{-1} \mathbf{Y}$ and $\mathbf{R}_{2}=\mathbf{E}^{T} \mathbf{P} \mathbf{C}^{+}$, where $\mathbf{C}^{+}$denotes the Moore-Penrose generalized inverse matrix of $\mathbf{C}$; that is, $\mathbf{C}^{+}=\mathbf{C}^{T}\left(\mathbf{C C}^{T}\right)^{-1}$.

Proof. Condition (27): according to Lemma 1, it is easy to prove that if condition (27) is satisfied, the eigenvalues of (A - LC) belong to $\mathrm{D}\left(\lambda_{2}, \tau_{2}\right)$. tion:

Condition (28): consider the following Lyapunov func-

$$
V(t)=\mathbf{e}_{x}^{T}(t) \mathbf{P} \mathbf{e}_{x}(t)+\frac{1}{\sigma} \mathbf{e}_{f}^{T}(t) \mathbf{e}_{f}(t)
$$

Then its time derivative is

$$
\begin{aligned}
\dot{V}(t)= & \dot{\mathbf{e}}_{x}^{T}(t) \mathbf{P} \mathbf{e}_{x}(t)+\mathbf{e}_{x}^{T}(t) \mathbf{P} \dot{\mathbf{e}}_{x}(t)+2 \frac{1}{\sigma} \mathbf{e}_{f}^{T}(t) \dot{\mathbf{e}}_{f}(t) \\
= & \mathbf{e}_{x}^{T}(t)\left(\mathbf{P}(\mathbf{A}-\mathbf{L C})+(\mathbf{A}-\mathbf{L C})^{T} \mathbf{P}\right) \mathbf{e}_{x}(t) \\
& +2 \mathbf{e}_{x}^{T}(t) \mathbf{P E} \mathbf{e}_{f}(t)+2 \mathbf{e}_{x}^{T}(t) \mathbf{P D} \boldsymbol{\omega}(t) \\
& +2 \frac{1}{\sigma} \mathbf{e}_{f}^{T}(t) \dot{\mathbf{e}}_{f}(t)
\end{aligned}
$$

Under assumption (24), 2(1/ $\sigma) \mathbf{e}_{f}^{T}(t) \dot{\mathbf{e}}_{f}(t)$ can be given as

$$
\begin{aligned}
2 \frac{1}{\sigma} \mathbf{e}_{f}^{T}(t) \dot{\mathbf{e}}_{f}(t) & \\
= & 2 \frac{1}{\sigma} \mathbf{e}_{f}^{T}(t) \mathbf{R}_{1} \mathbf{e}_{f}(t)-2 \frac{1}{\sigma} \mathbf{e}_{f}^{T}(t) \mathbf{R}_{2} \mathbf{C}\left(\dot{\mathbf{e}}_{x}(t)+\sigma \mathbf{e}_{x}(t)\right) \\
& -2 \frac{1}{\sigma} \mathbf{e}_{f}^{T}(t) \mathbf{R}_{1} \mathbf{f}(t)+2 \frac{1}{\sigma} \mathbf{e}_{f}^{T}(t) \dot{\mathbf{f}}(t) \\
= & 2 \frac{1}{\sigma} \mathbf{e}_{f}^{T}(t) \mathbf{R}_{1} \mathbf{e}_{f}(t)-2 \frac{1}{\sigma} \mathbf{e}_{f}^{T}(t) \mathbf{E}^{T} \mathbf{P}(\mathbf{A}-\mathbf{L C}) \mathbf{e}_{x}(t)
\end{aligned}
$$

$$
\begin{aligned}
& -2 \frac{1}{\sigma} \mathbf{e}_{f}^{T}(t) \mathbf{E}^{T} \mathbf{P E} e_{f}(t)-2 \frac{1}{\sigma} \mathbf{e}_{f}^{T}(t) \mathbf{E}^{T} \mathbf{P D} \boldsymbol{\omega}(t) \\
& -2 \mathbf{e}_{f}^{T}(t) \mathbf{E}^{T} \mathbf{P} \mathbf{e}_{x}(t)-2 \frac{1}{\sigma} \mathbf{e}_{f}^{T}(t) \mathbf{R}_{1} \mathbf{f}(t) \\
& +2 \frac{1}{\sigma} \mathbf{e}_{f}^{T}(t) \dot{\mathbf{f}}(t) .
\end{aligned}
$$

Substituting (31) into (30) yields

$$
\begin{aligned}
\dot{V}(t)= & \mathbf{e}_{x}^{T}(t)\left(\mathbf{P}(\mathbf{A}-\mathbf{L C})+(\mathbf{A}-\mathbf{L C})^{T} \mathbf{P}\right) \mathbf{e}_{x}(t) \\
& +2 \mathbf{e}_{x}^{T}(t) \mathbf{P D} \boldsymbol{\omega}(t)+2 \frac{1}{\sigma} \mathbf{e}_{f}^{T}(t) \mathbf{R}_{1} \mathbf{e}_{f}(t) \\
& -2 \frac{1}{\sigma} \mathbf{e}_{f}^{T}(t) \mathbf{E}^{T} \mathbf{P}(\mathbf{A}-\mathbf{L C}) \mathbf{e}_{x}(t) \\
& -2 \frac{1}{\sigma} \mathbf{e}_{f}^{T}(t) \mathbf{E}^{T} \mathbf{P E} e_{f}(t)-2 \frac{1}{\sigma} \mathbf{e}_{f}^{T}(t) \mathbf{E}^{T} \mathbf{P D} \boldsymbol{\omega}(t) \\
& -2 \frac{1}{\sigma} \mathbf{e}_{f}^{T}(t) \mathbf{R}_{1} \mathbf{f}(t)+2 \frac{1}{\sigma} \mathbf{e}_{f}^{T}(t) \dot{\mathbf{f}}(t) .
\end{aligned}
$$

Define

$$
J_{2}=\int_{0}^{T}\left[\frac{1}{\gamma_{2}} \widetilde{\mathbf{e}}^{T}(t) \widetilde{\mathbf{e}}(t)-\gamma_{2} \widetilde{\boldsymbol{\omega}}^{T}(t) \widetilde{\boldsymbol{\omega}}(t)\right] d t .
$$

Under the zero initial condition, that is, $V(0)=0$, it follows that

$$
\begin{aligned}
J_{2}= & \int_{0}^{T}\left[\frac{1}{\gamma_{2}} \widetilde{\mathbf{e}}^{T}(t) \widetilde{\mathbf{e}}(t)-\gamma_{2} \widetilde{\boldsymbol{\omega}}^{T}(t) \widetilde{\boldsymbol{\omega}}(t)+\dot{V}(t)\right] d t \\
& -(V(T)-V(0)) \\
\leq & \int_{0}^{T}\left[\frac{1}{\gamma_{2}} \widetilde{\mathbf{e}}^{T}(t) \widetilde{\mathbf{e}}(t)-\gamma_{2} \widetilde{\boldsymbol{\omega}}^{T}(t) \widetilde{\boldsymbol{\omega}}(t)+\dot{V}(t)\right] d t .
\end{aligned}
$$

Substituting (32) into (34) yields

$$
J_{2} \leq \int_{0}^{T}\left[\begin{array}{ll}
\widetilde{\mathbf{e}}^{T}(t) & \widetilde{\boldsymbol{\omega}}^{T}(t)
\end{array}\right] \boldsymbol{\Pi}\left[\begin{array}{c}
\widetilde{\mathbf{e}}(t) \\
\widetilde{\boldsymbol{\omega}}(t)
\end{array}\right] d t,
$$

where 


$$
\Pi=\left[\begin{array}{ccccc}
\frac{1}{\gamma_{2}} \mathbf{I}_{n}+\boldsymbol{\varphi}_{11} & -\frac{1}{\sigma}\left(\mathbf{A}^{T} \mathbf{P E}-\mathbf{C}^{T} \mathbf{Y}^{T} \mathbf{E}\right) & \mathbf{P D} & 0 & 0 \\
* & -2 \frac{1}{\sigma} \mathbf{E}^{T} \mathbf{P E}+\frac{1}{\gamma_{2}} \mathbf{I}_{r}+2 \frac{1}{\sigma} \mathbf{R}_{1} & -\frac{1}{\sigma} \mathbf{E}^{T} \mathbf{P D} & -\frac{1}{\sigma} \mathbf{R}_{1} & \frac{1}{\sigma} \mathbf{I}_{r} \\
* & * & -\gamma_{2} \mathbf{I}_{d} & 0 & 0 \\
* & * & * & -\gamma_{2} \mathbf{I}_{r} & 0 \\
* & * & * & * & -\gamma_{2} \mathbf{I}_{r}
\end{array}\right] .
$$

From above, it can be obtained that if LMI (28) holds, $\Pi<0$ holds with the application of Schur complement lemma. Further, one can obtain $J_{2}<0$; that is, $\|\widetilde{\mathbf{e}}(t)\|_{2}<$ $\gamma_{2}\|\widetilde{\boldsymbol{\omega}}(t)\|_{2}$. Meanwhile, condition (27) guarantees the existence of IAFEO. Therefore, if conditions (27) and (28) hold, the error dynamics (5) and (26) are stable and satisfy the $H_{\infty}$ performance index $\|\widetilde{\mathbf{e}}(t)\|_{2}<\gamma_{2}\|\widetilde{\boldsymbol{\omega}}(t)\|_{2}$. Since the measurement matrix $\mathbf{C}$ in this paper is of full row rank, its Moore-Penrose generalized inverse matrix is unique; that is, $\mathbf{C}^{+}=\mathbf{C}^{T}\left(\mathbf{C C}^{T}\right)^{-1}$. Therefore, the gain matrix $\mathbf{R}_{2}$ can be obtained by $\mathbf{R}_{2}=\mathbf{E}^{T} \mathbf{P C}$.

Remark 10. In Theorem 9, an IAFEO design method based on a new fault estimator model and multiobjective constraint is proposed. And in order to minimize $\widetilde{\mathbf{e}}(t)$ as much as possible, the minimum $H_{\infty}$ performance level $\gamma_{2}$ can be obtained by solving the convex optimization problem, that is, minimizing $\gamma_{2}$ subject to (27) and (28).

From the above design procedure of improved AFEA, the gain matrices $\mathbf{R}_{1}$ and $\mathbf{R}_{2}$ have been derived. However, fault estimator model (22) includes the time derivative of the output $\dot{\mathbf{y}}(t)$, which is not easily available in practical situations and may amplify the effect of the system noise on fault estimation performance. Therefore, in the following, the practical implementation of fault estimator will be presented.

Fault estimator (22) can be equivalently converted into

$$
\dot{\hat{\mathbf{f}}}(t)-\mathbf{R}_{2} \dot{\mathbf{e}}_{y}(t)=\mathbf{R}_{1} \widehat{\mathbf{f}}(t)+\sigma \mathbf{R}_{2} \mathbf{e}_{y}(t) .
$$

In order to eliminate the term $\dot{\mathbf{y}}(t)$ from (37), denote a new variable $\boldsymbol{\chi}(t)=\widehat{\mathbf{f}}(t)-\mathbf{R}_{2} \mathbf{e}_{y}(t)$ and it follows that

$$
\begin{aligned}
\dot{\chi}(t) & =\mathbf{R}_{1}\left(\chi(t)+\mathbf{R}_{2} \mathbf{e}_{y}(t)\right)+\sigma \mathbf{R}_{2} \mathbf{e}_{y}(t) \\
& =\mathbf{R}_{1} \boldsymbol{\chi}(t)+\left(\mathbf{R}_{1} \mathbf{R}_{2}+\sigma \mathbf{R}_{2}\right) \mathbf{e}_{y}(t)
\end{aligned}
$$

Further, one can obtain the on-line fault estimation

$$
\widehat{\mathbf{f}}(t)=\chi(t)+\mathbf{R}_{2} \mathbf{e}_{y}(t) .
$$

As can be seen in Theorem 9, $\sigma$ is given as a known scalar. But, in fact, how to choose the scalar $\sigma$ is a nontrivial problem. In this paper, it can be addressed in an approximate way based on the following theorem.
Theorem 11. Let $S$ be the set defined by

$$
S=\left\{\left(\mathbf{e}_{x}(t), \mathbf{e}_{f}(t)\right) \mid \lambda_{\min }(\mathbf{P})\left\|\mathbf{e}_{x}(t)\right\|_{2}^{2}+\frac{1}{\sigma}\left\|\mathbf{e}_{f}(t)\right\|_{2}^{2} \leq \frac{\varepsilon}{\alpha}\right\}
$$

where

$$
\begin{gathered}
\varepsilon=\left(\mu_{1}+\frac{\mu_{3}}{\sigma}\right) \omega_{0}^{2}+\frac{\mu_{4}}{\sigma} f_{0}^{2}+\frac{\mu_{5}}{\sigma} f_{1}^{2}, \\
\alpha=\frac{\min \left(b_{1}, b_{2}\right)}{\max \left[\lambda_{\max }(\mathbf{P}), 1 / \sigma\right]}, \\
b_{1}=\lambda_{\min }\left(\mathbf{Q}-\frac{1}{\mu_{1}} \mathbf{P D D}^{T} \mathbf{P}-\frac{\mu_{2}}{\sigma}(\mathbf{A}-\mathbf{L C})^{T}(\mathbf{A}-\mathbf{L C})\right)>0, \\
b_{2}=\lambda_{\min }\left(2 \frac{1}{\sigma} \mathbf{E}^{T} \mathbf{P E}-2 \frac{1}{\sigma} \mathbf{R}_{1}-\frac{1}{\sigma \mu_{2}} \mathbf{E}^{T} \mathbf{P P E}\right. \\
\left.-\frac{1}{\sigma \mu_{3}} \mathbf{E}^{T} \mathbf{P D D}^{T} \mathbf{P E}-\frac{1}{\sigma \mu_{4}} \mathbf{R}_{1}^{2}-\frac{1}{\sigma \mu_{5}} \mathbf{I}_{r}\right)>0,
\end{gathered}
$$

$\mu_{1}-\mu_{5}$ are positive scalars, and $\mathbf{Q}$ is a symmetric positive definite matrix, which satisfies condition (23); that is, $\mathbf{P}(\mathbf{A}-$ $\mathbf{L C})+(\mathbf{A}-\mathbf{L C})^{T} \mathbf{P}=-\mathbf{Q} . \mathbf{P}, \mathbf{L}$, and $\mathbf{R}_{1}$ can be calculated via Theorem 9. Then the IAFEO can guarantee that $\left(\mathbf{e}_{x}(t), \mathbf{e}_{f}(t)\right)$ converges to the set $S$ exponentially at a rate greater than $e^{-\alpha t}$.

Proof. By using Lemma 8 in (32), it is easy to obtain that

$$
\begin{aligned}
\dot{V}(t) \leq & -\mathbf{e}_{x}^{T}(t) \mathbf{Q} \mathbf{e}_{x}(t)+\frac{1}{\mu_{1}} \mathbf{e}_{x}^{T}(t) \mathbf{P D D} \mathbf{D}^{T} \mathbf{P} \mathbf{e}_{x}(t) \\
& +\mu_{1} \boldsymbol{\omega}^{T}(t) \boldsymbol{\omega}(t)+2 \frac{1}{\sigma} \mathbf{e}_{f}^{T}(t) \mathbf{R}_{1} \mathbf{e}_{f}(t) \\
& +\frac{1}{\sigma \mu_{2}} \mathbf{e}_{f}^{T}(t) \mathbf{E}^{T} \mathbf{P} \mathbf{P E} \mathbf{e}_{f}(t) \\
& +\frac{\mu_{2}}{\sigma} \mathbf{e}_{x}^{T}(t)(\mathbf{A}-\mathbf{L C})^{T}(\mathbf{A}-\mathbf{L C}) \mathbf{e}_{x}(t) \\
& -2 \frac{1}{\sigma} \mathbf{e}_{f}^{T}(t) \mathbf{E}^{T} \mathbf{P E} e_{f}(t) \\
& +\frac{1}{\sigma \mu_{3}} \mathbf{e}_{f}^{T}(t) \mathbf{E}^{T} \mathbf{P D D} \mathbf{D E}^{T} \mathbf{P E} \mathbf{e}_{f}(t)+\frac{\mu_{3}}{\sigma} \boldsymbol{\omega}^{T}(t) \boldsymbol{\omega}(t)
\end{aligned}
$$




$$
\begin{aligned}
& +\frac{1}{\sigma \mu_{4}} \mathbf{e}_{f}^{T}(t) \mathbf{R}_{1}^{2} \mathbf{e}_{f}(t)+\frac{\mu_{4}}{\sigma} \mathbf{f}^{T}(t) \mathbf{f}(t) \\
& +\frac{1}{\sigma \mu_{5}} \mathbf{e}_{f}^{T}(t) \mathbf{e}_{f}(t)+\frac{\mu_{5}}{\sigma} \dot{\mathbf{f}}^{T}(t) \dot{\mathbf{f}}(t)
\end{aligned}
$$

Since $\|\boldsymbol{\omega}(t)\|_{2} \leq \omega_{0},\|\mathbf{f}(t)\|_{2} \leq f_{0}$, and $\|\dot{\mathbf{f}}(t)\|_{2} \leq f_{1}$, one can further obtain

$$
\dot{V}(t) \leq-b_{1}\left\|\mathbf{e}_{x}\right\|_{2}^{2}-b_{2}\left\|\mathbf{e}_{f}\right\|_{2}^{2}+\varepsilon .
$$

From (29), one has

$$
\begin{aligned}
V & \leq \lambda_{\max }(\mathbf{P})\left\|\mathbf{e}_{x}\right\|_{2}^{2}+\frac{1}{\sigma}\left\|\mathbf{e}_{f}\right\|_{2}^{2} \\
& \leq \max \left[\lambda_{\max }(\mathbf{P}), \frac{1}{\sigma}\right]\left(\left\|\mathbf{e}_{x}\right\|_{2}^{2}+\left\|\mathbf{e}_{f}\right\|_{2}^{2}\right) .
\end{aligned}
$$

Substituting (44) into (43) yields

$$
\dot{V}(t) \leq-\alpha V(t)+\varepsilon,
$$

where the definitions of the scalars $\mu_{1}-\mu_{5}, b_{1}, b_{2}, \varepsilon$, and $\alpha$ in (42), (43), and (45) are given in (40).

Let $\bar{S}$ be the supplementary set of $S$. The following inequality holds as long as $\left(\mathbf{e}_{x}(t), \mathbf{e}_{f}(t)\right) \in \bar{S}$ :

$$
V(t) \geq \lambda_{\min }(\mathbf{P})\left\|\mathbf{e}_{x}(t)\right\|_{2}^{2}+\frac{1}{\sigma}\left\|\mathbf{e}_{f}(t)\right\|_{2}^{2} \geq \frac{\varepsilon}{\alpha} .
$$

From (45) and (46), it follows that if $\left(\mathbf{e}_{x}(t), \mathbf{e}_{f}(t)\right) \in \bar{S}$, the inequality $\dot{V}(t) \leq 0$ holds, which means that the trajectory of $\left(\mathbf{e}_{x}(t), \mathbf{e}_{f}(t)\right)$ inside $\bar{S}$ (i.e., outside the closed set $S$, since $\bar{S}$ is the supplementary set of $S$ ) is led to the set $S$ according to Lyapunov stability theory [36]. More specifically, the pair $\left(\mathbf{e}_{x}(t), \mathbf{e}_{f}(t)\right)$ is uniformly bounded and converges to the set $S$ exponentially at a rate greater than $e^{-\alpha t}$ due to (45).

From Theorem 9, the convergence set of $\left(\mathbf{e}_{x}(t), \mathbf{e}_{f}(t)\right)$ can be minimized according to $\|\widetilde{\mathbf{e}}(t)\|_{2}<\gamma_{2}\|\widetilde{\boldsymbol{\omega}}(t)\|_{2}$. So the research emphasis can be focused on increasing the convergence rate here. Due to $\alpha>0$, trying to obtain small $\alpha$ can increase the convergence rate $e^{-\alpha t}$. Since $\mu_{1}-\mu_{5}$ can be any positive scalars according to Lemma 8 , one can further obtain

$$
\begin{aligned}
& b_{1}^{\prime}=\lambda_{\text {min }}(\mathbf{Q})>b_{1}>0, \\
& b_{2}^{\prime}=\lambda_{\text {min }}\left(2 \frac{1}{\sigma} \mathbf{E}^{T} \mathbf{P E}-2 \frac{1}{\sigma} \mathbf{R}_{1}\right)>b_{2}>0 .
\end{aligned}
$$

Then imposing, for example, $\lambda_{\max }(\mathbf{P})>1 / \sigma$, one gets

$$
\alpha=\frac{\min \left(b_{1}^{\prime}, b_{2}^{\prime}\right)}{\lambda_{\max }(\mathbf{P})} .
$$

Therefore from a practical point of view, by using the Matlab LMI toolbox to solve the convex optimization problem in Theorem 9, a computational approach could be used to choose an appropriate $\sigma$ such that $\min \left(b_{1}^{\prime}, b_{2}^{\prime}\right)$ becomes small and $\lambda_{\max }(\mathbf{P})$ becomes large, which can increase the convergence rate $e^{-\alpha t}$.

\section{Fault-Tolerant Controller Design}

Compared with the AFEA, the improved AFEA evidently improves the performance of fault estimation in accordance with the above theoretical analysis. Therefore, the faulttolerant controller designed in this section is based on the improved AFEA. Considering that the state variables of most practical systems are generally unmeasurable, an OSFFTC is designed to stabilize the closed-loop system in the presence of faults.

On the basis of the on-line state and fault estimation, the feedback fault-tolerant controller is constructed as

$$
\mathbf{u}(t)=-\mathbf{K} \widehat{\mathbf{x}}(t)-\mathbf{B}^{*} \mathbf{E} \widehat{\mathbf{f}}(t),
$$

where $\mathbf{K} \in \mathbf{R}^{m \times n}$ is the OSFFTC gain matrix and the definition of $\mathbf{B}^{*} \in \mathbf{R}^{m \times n}$ will be given later.

Then, the design of the proposed fault-tolerant controller makes use of the following one lemma and one assumption.

Lemma 12 (see [36]). The OSFFTC gain matrix $\mathbf{K}$ can be chosen such that $\mathbf{A}-\mathbf{B K}$ is stable due to the controllability of (A, B).

Remark 13. As can be easily seen from Lemma 12, the fact that (A, B) is controllable is a sufficient condition for the existence of $\mathbf{K}$. On the other hand, if $\mathbf{A}-\mathbf{B K}$ is stable, given $\mathbf{W}=\mathbf{W}^{T}>$ 0 , there exists a unique stabilizing solution $\mathbf{H}=\mathbf{H}^{T}>0$ of the following Lyapunov equation:

$$
(\mathbf{A}-\mathbf{B K})^{T} \mathbf{H}+\mathbf{H}(\mathbf{A}-\mathbf{B K})=-\mathbf{W} .
$$

Assumption 14 (see [24]). Consider $\operatorname{rank}(\mathbf{B}, \mathbf{E})=\operatorname{rank}(\mathbf{B})$.

Remark 15. Assumption 14 is always satisfied when $\operatorname{rank}(\mathbf{B})=n$. When $\operatorname{rank}(\mathbf{B})<n$, it is satisfied only for specific fault distributions. Therefore, it can be seen that only faults whose effect belongs to the actuation space, that is, $\operatorname{span}(\mathbf{B})$, are considered, where $\operatorname{span}(\mathbf{B})$ denotes the vector space spanned by the column vectors of $\mathbf{B}$. Namely, $\operatorname{rank}(\mathbf{B}, \mathbf{E})=\operatorname{rank}(\mathbf{B}) \Leftrightarrow \operatorname{span}(\mathbf{E}) \subseteq \operatorname{span}(\mathbf{B})$, which is equivalent to the existence of $\mathbf{B}^{*}$ such that

$$
\left(\mathbf{I}_{n}-\mathbf{B B}^{*}\right) \mathbf{E}=0 .
$$

Considering that $\mathbf{B}^{*}$ appears in (51), how to choose $\mathbf{B}^{*}$ is introduced here. $\operatorname{span}(\mathbf{E}) \subseteq \operatorname{span}(\mathbf{B})$ is equivalent to the fact that there exists a nonzero matrix $\overline{\mathbf{E}} \in \mathbf{R}^{m \times r}$ such that $\mathbf{E}=\mathbf{B} \overline{\mathbf{E}}$. Because $\mathbf{B}$ is of full column rank, its MoorePenrose generalized inverse matrix is unique; that is, $\mathbf{B}^{+}=$ $\left(\mathbf{B}^{T} \mathbf{B}\right)^{-1} \mathbf{B}^{T}$. It follows that

$$
\left(\mathbf{I}_{n}-\mathbf{B B}^{+}\right) \mathbf{B}=\mathbf{B}-\mathbf{B}\left(\mathbf{B}^{+} \mathbf{B}\right)=\mathbf{B}-\mathbf{B}=0 .
$$

Further, one gets

$$
\left(\mathbf{I}_{n}-\mathbf{B B}^{+}\right) \mathbf{E}=\left(\mathbf{I}_{n}-\mathbf{B B}^{+}\right) \mathbf{B} \overline{\mathbf{E}}=0 \cdot \overline{\mathbf{E}}=0 .
$$

So it satisfies (51). Therefore, $\mathbf{B}^{*}$ can be chosen as $\mathbf{B}^{+}$; that is, $\mathbf{B}^{*}=\left(\mathbf{B}^{T} \mathbf{B}\right)^{-1} \mathbf{B}^{T}$. 
According to the above description, all the poles of system (1) can be arbitrarily assigned by state feedback control since $(\mathbf{A}, \mathbf{B})$ is controllable. Therefore, the proposed design method of FTC is to assign all poles of the closed-loop system to a special region in the left-half side of the complex plane to make the system obtain a desired degree of stability.

Theorem 16. Under Lemma 12 and Assumption 14, system (1) and (2) is stabilized by the OSFFTC (49).

Proof. Applying the control (49) to system (1) results in the following closed-loop dynamics:

$$
\begin{aligned}
\dot{\mathbf{x}}(t)= & \mathbf{A x}(t)-\mathbf{B K} \widehat{\mathbf{x}}(t)-\mathbf{B B}^{*} \mathbf{E} \widehat{\mathbf{f}}(t)+\mathbf{E} \mathbf{f}(t)+\mathbf{D} \boldsymbol{\omega}(t) \\
= & (\mathbf{A}-\mathbf{B K}) \mathbf{x}(t)+\mathbf{B K} \mathbf{e}_{x}(t)+\left(\mathbf{I}_{n}-\mathbf{B B}^{*}\right) \mathbf{E} \widehat{\mathbf{f}}(t) \\
& +\mathbf{E e}_{f}(t)+\mathbf{D} \boldsymbol{\omega}(t),
\end{aligned}
$$

where $\mathbf{K}$ is the state feedback control gain matrix such that $\mathbf{A}-\mathbf{B K}$ is stable.

Substituting (51) into (54) yields

$$
\dot{\mathbf{x}}(t)=(\mathbf{A}-\mathbf{B K}) \mathbf{x}(t)+\mathbf{B K} \mathbf{e}_{x}(t)+\mathbf{E} \mathbf{e}_{f}(t)+\mathbf{D} \boldsymbol{\omega}(t) .
$$

Consider the Lyapunov function

$$
V(t)=\mathbf{e}_{x}^{T}(t) \mathbf{P} \mathbf{e}_{x}(t)+\frac{1}{\sigma} \mathbf{e}_{f}^{T}(t) \mathbf{e}_{f}(t)+\mathbf{x}^{T}(t) \mathbf{H} \mathbf{x}(t)
$$

where $\mathbf{P}>0$ is calculated by Theorem $9, \sigma$ is chosen in Section 4 , and $\mathbf{H}>0$ is the unique stabilizing solution of (50) for a given $\mathbf{W}$.

From (32) and (55), the time derivative of $V(t)$ is

$$
\begin{aligned}
\dot{V}(t)= & \mathbf{e}_{x}^{T}(t)\left(\mathbf{P}(\mathbf{A}-\mathbf{L C})+(\mathbf{A}-\mathbf{L} \mathbf{C})^{T} \mathbf{P}\right) \mathbf{e}_{x}(t) \\
& +2 \mathbf{e}_{x}^{T}(t) \mathbf{P D} \boldsymbol{\omega}(t)+2 \frac{1}{\sigma} \mathbf{e}_{f}^{T}(t) \mathbf{R}_{1} \mathbf{e}_{f}(t) \\
& -2 \frac{1}{\sigma} \mathbf{e}_{f}^{T}(t) \mathbf{E}^{T} \mathbf{P}(\mathbf{A}-\mathbf{L C}) \mathbf{e}_{x}(t) \\
& -2 \frac{1}{\sigma} \mathbf{e}_{f}^{T}(t) \mathbf{E}^{T} \mathbf{P E} e_{f}(t)-2 \frac{1}{\sigma} \mathbf{e}_{f}^{T}(t) \mathbf{E}^{T} \mathbf{P D} \boldsymbol{\omega}(t) \\
& -2 \frac{1}{\sigma} \mathbf{e}_{f}^{T}(t) \mathbf{R}_{1} \mathbf{f}(t)+2 \frac{1}{\sigma} \mathbf{e}_{f}^{T}(t) \dot{\mathbf{f}}(t) \\
& +\mathbf{x}^{T}(t)\left((\mathbf{A}-\mathbf{B K})^{T} \mathbf{H}+\mathbf{H}(\mathbf{A}-\mathbf{B K})\right) \mathbf{x}(t) \\
& +2 \mathbf{x}^{T}(t) \mathbf{H B K} \mathbf{e}_{x}(t)+2 \mathbf{x}^{T}(t) \mathbf{H E} \mathbf{e}_{f}(t) \\
& +2 \mathbf{x}^{T}(t) \mathbf{H D} \boldsymbol{\omega}(t) .
\end{aligned}
$$

According to Lemma 8, (57) can be converted to

$$
\begin{aligned}
\dot{V}(t) \leq & -\mathbf{e}_{x}^{T}(t) \mathbf{Q} \mathbf{e}_{x}(t)+\frac{1}{\mu_{1}} \mathbf{e}_{x}^{T}(t) \mathbf{P D D} \mathbf{D}^{T} \mathbf{P} \mathbf{e}_{x}(t) \\
& +\mu_{1} \boldsymbol{\omega}^{T}(t) \boldsymbol{\omega}(t)+2 \frac{1}{\sigma} \mathbf{e}_{f}^{T}(t) \mathbf{R}_{1} \mathbf{e}_{f}(t) \\
& +\frac{1}{\sigma \mu_{2}} \mathbf{e}_{f}^{T}(t) \mathbf{E}^{T} \mathbf{P} \mathbf{P E} \mathbf{e}_{f}(t) \\
& +\frac{\mu_{2}}{\sigma} \mathbf{e}_{x}^{T}(t)(\mathbf{A}-\mathbf{L C})^{T}(\mathbf{A}-\mathbf{L C}) \mathbf{e}_{x}(t) \\
& -2 \frac{1}{\sigma} \mathbf{e}_{f}^{T}(t) \mathbf{E}^{T} \mathbf{P E} e_{f}(t)+\frac{1}{\sigma \mu_{3}} \mathbf{e}_{f}^{T}(t) \mathbf{E}^{T} \mathbf{P D D} \mathbf{D}^{T} \mathbf{P E} \mathbf{e}_{f}(t) \\
& +\frac{\mu_{3}}{\sigma} \boldsymbol{\omega}^{T}(t) \boldsymbol{\omega}(t) \\
& +\frac{1}{\sigma \mu_{4}} \mathbf{e}_{f}^{T}(t) \mathbf{R}_{1}^{2} \mathbf{e}_{f}(t)+\frac{\mu_{4}}{\sigma} \mathbf{f}^{T}(t) \mathbf{f}(t) \\
& +\frac{1}{\sigma \mu_{5}} \mathbf{e}_{f}^{T}(t) \mathbf{e}_{f}(t)+\frac{\mu_{5}}{\sigma} \dot{\mathbf{f}}^{T}(t) \dot{\mathbf{f}}(t) \\
& +\frac{1}{\mu_{8}} \mathbf{x}^{T}(t) \mathbf{H D D} \mathbf{H}^{T} \mathbf{x}(t)+\mu_{8} \boldsymbol{\omega}^{T}(t) \boldsymbol{\omega}(t) \\
& -\mathbf{x}^{T}(t) \mathbf{W} \mathbf{x}(t)+\frac{1}{\mu_{6}} \mathbf{x}^{T}(t) \mathbf{H B K K} \mathbf{K}^{T} \mathbf{B}^{T} \mathbf{H} \mathbf{x}(t) \\
& +\mu_{6} \mathbf{e}_{x}^{T}(t) \mathbf{e}_{x}(t) \\
& +\frac{1}{\mu_{7}} \mathbf{x}^{T}(t) \mathbf{H E} \mathbf{E}^{T} \mathbf{H} \mathbf{x}(t)+\mu_{7} \mathbf{e}_{f}^{T}(t) \mathbf{e}_{f}(t) \\
& \\
&
\end{aligned}
$$

where $\mu_{1}-\mu_{8}$ are positive scalars, $\mathbf{W}$ is given $\mathbf{W}=\mathbf{W}^{T}>0$, and $\mathbf{Q}, \mathbf{L}$, and $\mathbf{R}_{1}$ are the same as those in Theorem 11 .

Then, one can further obtain

$$
\dot{V}(t) \leq-c_{1}\|\mathbf{x}(t)\|_{2}^{2}-c_{2}\left\|\mathbf{e}_{x}(t)\right\|_{2}^{2}-c_{3}\left\|\mathbf{e}_{f}(t)\right\|_{2}^{2}+\beta
$$

where

$$
\begin{gathered}
c_{1}=\lambda_{\min }\left(\mathbf{W}-\mathbf{H}\left(\frac{\mathbf{B} \mathbf{K} \mathbf{K}^{T} \mathbf{B}^{T}}{\mu_{6}}+\frac{\mathbf{E E}^{T}}{\mu_{7}}+\frac{\mathbf{D D}^{T}}{\mu_{8}}\right) \mathbf{H}\right), \\
c_{2}=\lambda_{\min }\left(\mathbf{Q}-\mu_{6} \mathbf{I}_{n}-\frac{1}{\mu_{1}} \mathbf{P D D}^{T} \mathbf{P}\right. \\
\left.-\frac{\mu_{2}}{\sigma}(\mathbf{A}-\mathbf{L C})^{T}(\mathbf{A}-\mathbf{L C})\right), \\
c_{3}=\lambda_{\min }\left(2 \frac{1}{\sigma} \mathbf{E}^{T} \mathbf{P E}-2 \frac{1}{\sigma} \mathbf{R}_{1}-\frac{1}{\sigma \mu_{2}} \mathbf{E}^{T} \mathbf{P P E}\right. \\
-\frac{1}{\sigma \mu_{3}} \mathbf{E}^{T} \mathbf{P D D} \mathbf{D}^{T} \mathbf{P E}-\frac{1}{\sigma \mu_{4}} \mathbf{R}_{1}^{2}
\end{gathered}
$$




$$
\begin{gathered}
\left.-\left(\mu_{7}+\frac{1}{\sigma \mu_{5}}\right) \mathbf{I}_{r}\right), \\
\beta=\left(\mu_{1}+\mu_{8}+\frac{\mu_{3}}{\sigma}\right) \omega_{0}^{2}+\frac{\mu_{4}}{\sigma} f_{0}^{2}+\frac{\mu_{5}}{\sigma} f_{1}^{2} .
\end{gathered}
$$

Since $\mathbf{x}(t)$ is generally unmeasurable, substitute $\widehat{\mathbf{x}}(t)$ for $\mathbf{x}(t)$ by using the following inequalities, where the state estimation $\widehat{\mathbf{x}}(t)$ can be obtained by IAFEO, that is, $\widehat{\mathbf{x}}(t)$ being known:

$$
\begin{aligned}
\|\mathbf{x}(t)\|_{2} & =\left\|\widehat{\mathbf{x}}(t)+\mathbf{e}_{x}(t)\right\|_{2} \geq\|\widehat{\mathbf{x}}(t)\|_{2}-\left\|\mathbf{e}_{x}(t)\right\|_{2}, \\
\|\mathbf{x}(t)\|_{2}^{2} & \geq\left(\|\widehat{\mathbf{x}}(t)\|_{2}-\left\|\mathbf{e}_{x}(t)\right\|_{2}\right)^{2} \\
& =\|\widehat{\mathbf{x}}(t)\|_{2}^{2}+\left\|\mathbf{e}_{x}(t)\right\|_{2}^{2}-2\left\|\mathbf{e}_{x}(t)\right\|_{2}\|\widehat{\mathbf{x}}(t)\|_{2} .
\end{aligned}
$$

From Theorem 11, $\left\|\mathbf{e}_{x}\right\|_{2}$ is uniformly ultimately bounded and satisfies

$$
\left\|\mathbf{e}_{x}(t)\right\|_{2} \leq \mathbf{e}_{0},
$$

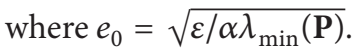

Substituting (62) into (61) yields

$$
\|\mathbf{x}(t)\|_{2}^{2} \geq\|\widehat{\mathbf{x}}(t)\|_{2}^{2}+\left\|\mathbf{e}_{x}(t)\right\|_{2}^{2}-2 e_{0}\|\widehat{\mathbf{x}}(t)\|_{2} .
$$

From (59), it follows that $\dot{V}(t)<0$ for

$$
c_{1}\|\mathbf{x}(t)\|_{2}^{2}+c_{2}\left\|\mathbf{e}_{x}(t)\right\|_{2}^{2}+c_{3}\left\|\mathbf{e}_{f}(t)\right\|_{2}^{2}>\beta .
$$

Substituting (63) into (64) yields

$$
\begin{aligned}
& c_{1}\|\mathbf{x}(t)\|_{2}^{2}+c_{2}\left\|\mathbf{e}_{x}(t)\right\|_{2}^{2}+c_{3}\left\|\mathbf{e}_{f}(t)\right\|_{2}^{2} \\
& \geq c_{1}\left(\|\widehat{\mathbf{x}}(t)\|_{2}^{2}+\left\|\mathbf{e}_{x}(t)\right\|_{2}^{2}-2 e_{0}\|\widehat{\mathbf{x}}(t)\|_{2}\right) \\
&+c_{2}\left\|\mathbf{e}_{x}(t)\right\|_{2}^{2}+c_{3}\left\|\mathbf{e}_{f}(t)\right\|_{2}^{2} \\
&= c_{1}\|\widehat{\mathbf{x}}(t)\|_{2}^{2}-2 c_{1} e_{0}\|\widehat{\mathbf{x}}(t)\|_{2}+\left(c_{1}+c_{2}\right)\left\|\mathbf{e}_{x}(t)\right\|_{2}^{2} \\
&+c_{3}\left\|\mathbf{e}_{f}(t)\right\|_{2}^{2} .
\end{aligned}
$$

Therefore, if the following conditions hold, inequality (64) is satisfied:

$$
\begin{gathered}
c_{1}\|\widehat{\mathbf{x}}(t)\|_{2}^{2}-2 c_{1} e_{0}\|\widehat{\mathbf{x}}(t)\|_{2}>\beta, \\
c_{1}+c_{2}>0, \\
c_{3}>0 .
\end{gathered}
$$

From (66), one can further obtain

$$
c_{1}\|\widehat{\mathbf{x}}(t)\|_{2}\left(\|\widehat{\mathbf{x}}(t)\|_{2}-2 e_{0}\right)>\beta .
$$

So it can be divided into two situations. One is that if $\|\widehat{\mathbf{x}}(t)\|_{2}>2 e_{0}$, condition (66) is equivalent to choosing $\mu_{1}$ and $\mu_{3}-\mu_{8}$ in $c_{1}$ and $\beta$ such that

$$
c_{1}>\frac{\beta}{\|\widehat{\mathbf{x}}(t)\|_{2}\left(\|\widehat{\mathbf{x}}(t)\|_{2}-2 e_{0}\right)} .
$$

The other one is that if $\|\widehat{\mathbf{x}}(t)\|_{2}<2 e_{0}$, the objective is to choose the corresponding constants such that

$$
c_{1}<\frac{\beta}{\|\widehat{\mathbf{x}}(t)\|_{2}\left(\|\widehat{\mathbf{x}}(t)\|_{2}-2 e_{0}\right)} .
$$

Therefore, since $\mu_{1}-\mu_{8}$ can be any positive scalars according to Lemma 8, it is easy to show that by choosing these constants conditions (66)-(68) can always hold. Further, $\dot{V}(t)<0$, which means that the feedback FTC (49) can guarantee that the closed-loop system is stable in the presence of faults.

Remark 17. As can be seen from Theorem 16, if $\mathbf{A}-\mathbf{B K}$ is stable, the OSFFTC can guarantee the stability of the faulty system. Therefore, given a set of self-conjugate closed-loop pole locations in the left-half side of the complex plane, the feedback control gain matrix $\mathbf{K}$ can be calculated.

\section{Simulation Analysis and Results}

6.1. System Description. In this section, the lateral motion model of a light aircraft borrowed from [30] is given in the state space formulation as follows, which is considered to illustrate the effectiveness of the design method in this paper:

$$
\begin{aligned}
& \dot{\mathbf{x}}(t)=\mathbf{A x}(t)+\mathbf{B u}(t)+\mathbf{E} \mathbf{f}(t), \\
& \mathbf{y}(t)=\mathbf{C x}(t),
\end{aligned}
$$

where $\mathbf{x}(t)=\left[V_{s}, r, q, \theta\right]$ and $\mathbf{u}(t)=\left[\delta_{r}, \delta_{a}\right]$, which are the sideslip velocity $(\mathrm{m} / \mathrm{s})$, roll rate $(\mathrm{rad} / \mathrm{s})$, yaw rate $(\mathrm{rad} / \mathrm{s})$, and roll angle ( $\mathrm{rad})$ and the rudder angle $\delta_{r}(\mathrm{rad})$ and aileron angle $\delta_{a}$ (rad). The model parameter matrices are given as follows:

$$
\begin{gathered}
\mathbf{A}=\left[\begin{array}{cccc}
-0.2770 & 0 & -32.9000 & 9.8100 \\
-0.1030 & -8.3250 & 3.7500 & 0 \\
0.3650 & 0 & -0.6390 & 0 \\
0 & 1 & 0 & 0
\end{array}\right], \\
\mathbf{B}=\left[\begin{array}{cc}
-5.4320 & 0 \\
0 & -28.6400 \\
-9.4900 & 0 \\
0 & 0
\end{array}\right], \quad \mathbf{C}=\left[\begin{array}{llll}
0 & 0 & 1 & 0 \\
0 & 0 & 0 & 1
\end{array}\right] .
\end{gathered}
$$

It is easy to verify that the pair $(\mathbf{A}, \mathbf{B})$ is controllable and the pair $(\mathbf{A}, \mathbf{C})$ is observable. In this particular situation, actuator faults are considered, which means that faults generally occur in the input channel and so it is assumed that $\mathbf{E}=\mathbf{B}$. According to the actual situation, appropriate interference $\boldsymbol{\omega}(t)$ (including external disturbance and model uncertainties) is added into the system and it is assumed that the disturbance distribution matrix is $\mathbf{D}=$ $\left[\begin{array}{llll}0.01 & 0.01 & 0.01 & 0.01\end{array}\right]^{T}$.

6.2. Parameter Calculation. By constructing augmented system (11), it is easy to show that the pair $(\widetilde{\mathbf{A}}, \widetilde{\mathbf{C}})$ is completely 
observable, which means that AFEO exists. And the IAFEO also exists for the observability of the pair $(\mathbf{A}, \mathbf{C})$.
Then the augmented matrices of (11) can be constructed as

$$
\begin{aligned}
& \widetilde{\mathbf{A}}=\left[\begin{array}{cccccc}
-0.2770 & 0 & -32.9000 & 9.8100 & -5.4320 & 0 \\
-0.1030 & -8.3250 & 3.7500 & 0 & 0 & -28.6400 \\
0.3650 & 0 & -0.6390 & 0 & -9.4900 & 0 \\
0 & 1 & 0 & 0 & 0 & 0 \\
0 & 0 & 0 & 0 & 1 & 0 \\
0 & 0 & 0 & 0 & 0 & 1
\end{array}\right] \text {, } \\
& \widetilde{\mathbf{C}}=\left[\begin{array}{llllll}
0 & 0 & 1 & 0 & 0 & 0 \\
0 & 0 & 0 & 1 & 0 & 0
\end{array}\right], \quad \widetilde{\mathbf{D}}=\left[\begin{array}{ccccc}
0.01 & 0 & 0 & 0 & 0 \\
0.01 & 0 & 0 & 0 & 0 \\
0.01 & 0 & 0 & 0 & 0 \\
0.01 & 0 & 0 & 0 & 0 \\
0 & -1 & 0 & 1 & 0 \\
0 & 0 & -1 & 0 & 1
\end{array}\right]
\end{aligned}
$$

According to Theorem 3, solving condition (14) with the regional pole constraint $D(-10,9.8)$ and condition (15) based on the mincx function in the Matlab LMI toolbox, one obtains the minimum value $\gamma_{1}=0.4614$ with

$$
\widetilde{\mathbf{Y}}=10^{5} \times\left[\begin{array}{cc}
-6.1956 & 1.8523 \\
0.0000 & -0.0006 \\
3.5497 & -1.0603 \\
-0.0005 & 0.0225 \\
0.0015 & -0.0001 \\
0.0003 & 0.0002
\end{array}\right] \text {. }
$$

So by solving $\widetilde{\mathbf{L}}=\widetilde{\mathbf{P}}^{-1} \widetilde{\mathbf{Y}}$, the AFEO gain matrices are

$$
\begin{aligned}
\mathbf{L} & =\left[\begin{array}{cc}
-16.5445 & 9.8034 \\
3.6951 & 300.9384 \\
28.4217 & 0.0004 \\
0.0010 & 29.8634
\end{array}\right], \\
\mathbf{R} & =\left[\begin{array}{cc}
-26.7509 & -0.0094 \\
-0.0009 & -129.2019
\end{array}\right] .
\end{aligned}
$$

According to Theorem 9, by choosing an appropriate value $\sigma=1$, which allows increasing the convergence rate of $\left(\mathbf{e}_{x}(t), \mathbf{e}_{f}(t)\right)$ according to Theorem 11, and solving conditions (27) and (28) with the same regional pole constraint $D(-10,9.8)$, one can obtain the minimum value $\gamma_{2}=0.001$. So it can be checked that improved AFEA gives the optimal value 0.001 which is smaller than the one given by AFEA, thus illustrating the superiority of improved AFEA. In order to prevent obtaining the overlarge observer gain matrices, the
$H_{\infty}$ performance level $\gamma_{2}$ is set $\gamma_{2}=0.04$ and one can further obtain from Theorem 9

$$
\begin{gathered}
\mathbf{P}=10^{3} \times\left[\begin{array}{cccc}
1.1556 & -0.0799 & -0.6013 & -0.6495 \\
-0.0799 & 0.0277 & -0.0135 & 0.1518 \\
-0.6013 & -0.0135 & 0.8124 & 0.0708 \\
-0.6495 & 0.1518 & 0.0708 & 1.4467
\end{array}\right], \\
\mathbf{Y}=10^{4} \times\left[\begin{array}{cc}
-3.8143 & 1.1161 \\
0.2540 & -0.0580 \\
2.0783 & -0.5824 \\
2.2440 & -0.0499
\end{array}\right] \\
\mathbf{R}_{1}=\left[\begin{array}{cc}
0.0061 & -0.0135 \\
-0.0135 & -0.0396
\end{array}\right]
\end{gathered}
$$

Since $\mathbf{C}$ is of full row rank, its Moore-Penrose generalized inverse matrix is unique as

$$
\mathbf{C}^{+}=\mathbf{C}^{T}\left(\mathbf{C C}^{T}\right)^{-1}=\left[\begin{array}{ll}
0 & 0 \\
0 & 0 \\
1 & 0 \\
0 & 1
\end{array}\right]
$$


Therefore, the IAFEO gain matrices $\mathbf{L}$ and $\mathbf{R}_{2}$ are

$$
\begin{gathered}
\mathbf{L}=\mathbf{P}^{-1} \mathbf{Y}=\left[\begin{array}{cc}
-31.3743 & 13.1206 \\
-12.0862 & -29.4574 \\
1.9337 & 1.3059 \\
2.5989 & 8.5740
\end{array}\right], \\
\mathbf{R}_{2}=\mathbf{E}^{T} \mathbf{P C}^{+}=\left[\begin{array}{cc}
-12.3937 & -0.0067 \\
-0.3380 & -74.9894
\end{array}\right] .
\end{gathered}
$$

From Remark 17, the desired self-conjugate closed-loop pole locations, which make $\mathbf{A}-\mathbf{B K}$ stable, are not unique. So choosing a set of closed-loop poles

$$
\mathbf{p}=\left[\begin{array}{llll}
-1.5+7.8 i-1.5-7.8 i & -28 & -51
\end{array}\right],
$$

the OSFFTC gain matrix $\mathbf{K}$ can be calculated

$$
\mathbf{K}=\left[\begin{array}{cccc}
1.4616 & 0.1932 & -7.0651 & 17.0663 \\
1.1713 & -0.4766 & -2.0597 & 5.3483
\end{array}\right]
$$

Then, choosing $\mathbf{W}=0.5 \mathbf{I}_{4}$ and solving (49), $\mathbf{H}$ can be obtained as

$$
\mathbf{H}=\left[\begin{array}{cccc}
0.1863 & 0.0486 & -0.2319 & 0.7148 \\
0.0486 & 0.0354 & -0.0683 & 0.6013 \\
-0.2319 & -0.0683 & 0.3037 & -1.0227 \\
0.7148 & 0.6013 & -1.0227 & 15.0067
\end{array}\right] \text {. }
$$

From Remark 15, B* can be computed as

$$
\mathbf{B}^{*}=\left(\mathbf{B}^{T} \mathbf{B}\right)^{-1} \mathbf{B}^{T}=\left[\begin{array}{cccc}
-0.0454 & 0 & -0.0794 & 0 \\
0 & -0.0349 & 0 & 0
\end{array}\right] \text {. }
$$

It follows that

$$
\mathbf{B}^{*} \mathbf{E}=\left[\begin{array}{ll}
1 & 0 \\
0 & 1
\end{array}\right]
$$

Therefore, the OSFFTC gain matrices $\mathbf{K}$ and $\mathbf{B}^{*} \mathbf{E}$ can be obtained.

6.3. Simulation Results. In simulation, taking the sampling time $T=0.01 \mathrm{~s}$, the system is subject to the input $\mathbf{u}(t)=$ $\left[\begin{array}{ll}6 & 6\end{array}\right]^{T}$ and initial value $\mathbf{x}(0)=\left[\begin{array}{llll}0 & 0 & 0 & 0\end{array}\right]^{T}$. In order to assess the robustness of the proposed algorithm, the external disturbance (i.e., wind turbulence acting on aircraft) is supposed to be a band-limited white noise with power 0.01 and random model uncertainties of $\mathbf{A}$ and $\mathbf{B}$ are involved in simulation, where modeling error matrices are assumed to be

$$
\Delta \mathbf{A}=\left[\begin{array}{cccc}
0 & 0.01 & 0 & 0 \\
0.02 & 0.01 & 0.01 & 0.01 \\
0 & 0 & 0.01 & 0 \\
0.02 & 0.01 & 0.01 & 0.01
\end{array}\right], \quad \Delta \mathbf{B}=\left[\begin{array}{cc}
0.01 & 0.02 \\
0 & 0 \\
0.01 & 0.02 \\
0 & 0
\end{array}\right] \text {. }
$$

In this paper, the 1st derivatives of the fault $\mathbf{f}(t)$ are in existence. Although many actuator faults lead to abrupt changes, in practice, actuator faults can also be caused by the degradation of components and behave as slow changes. Therefore, both the abrupt fault and the slow-varying fault are considered in the following.

(1) Single Fault. Assume that single abrupt fault $\mathbf{f}(t)=$ $\left[\begin{array}{ll}\mathbf{f}_{1}(t) & \mathbf{f}_{2}(t)\end{array}\right]^{T}$ is created as

$$
\begin{aligned}
& \mathbf{f}_{1}(t)= \begin{cases}0, & 0 \mathrm{~s} \leq t<3 \mathrm{~s}, \\
6\left(1-e^{-2(t-3)}\right), & 3 \mathrm{~s} \leq t<10 \mathrm{~s},\end{cases} \\
& \mathbf{f}_{2}(t)=0,
\end{aligned}
$$

which could be interpreted as a large deviation in the rudder angle.

Figures 2-4, respectively, illustrate the estimation of single abrupt fault using conventional algorithm [23], AFEA, and improved AFEA.

Assume that single slow-varying fault $\mathbf{f}(t)=$ $\left[\begin{array}{ll}\mathbf{f}_{1}(t) & \mathbf{f}_{2}(t)\end{array}\right]^{T}$ is created as

$$
\begin{aligned}
& \mathbf{f}_{1}(t)=0 \\
& \mathbf{f}_{2}(t)= \begin{cases}0, & 0 \mathrm{~s} \leq t<3 \mathrm{~s}, \\
6 \sin (t-3), & 3 \mathrm{~s} \leq t<10 \mathrm{~s}\end{cases}
\end{aligned}
$$

which could be interpreted as a gradual change in the aileron angle.

Figures 5-7, respectively, illustrate the estimation of single slow-varying fault using conventional algorithm [23], AFEA, and improved AFEA.

(2) Combined Fault. Assume that combined fault $\mathbf{f}(t)=$ $\left[\begin{array}{ll}\mathbf{f}_{1}(t) & \mathbf{f}_{2}(t)\end{array}\right]^{T}$ is created as

$$
\begin{gathered}
\mathbf{f}_{1}(t)= \begin{cases}0, & 0 \mathrm{~s} \leq t<2 \mathrm{~s}, \\
5 \sin (t-2), & 2 \mathrm{~s} \leq t<10 \mathrm{~s},\end{cases} \\
\mathbf{f}_{2}(t)= \begin{cases}0, & 0 \mathrm{~s} \leq t<5 \mathrm{~s}, \\
5\left(1-e^{-3(t-5)}\right), & 5 \mathrm{~s} \leq t<10 \mathrm{~s},\end{cases}
\end{gathered}
$$

where $\mathbf{f}_{1}(t)$ is a slow-varying fault and $\mathbf{f}_{2}(t)$ is an abrupt fault, which means a gradual change occurs in the rudder angle and a large deviation happens to the aileron angle. The simulation results for fault estimation using conventional algorithm [23], AFEA, and improved AFEA are shown, respectively, in Figures 8-10.

Under the different fault cases, Figures 11-13, respectively, illustrate the system output responses.

From the above simulation results, it can be concluded that, for the different types of faults, asymptotic convergence of fault estimation error can be achieved using two algorithms in this paper, and compared with conventional algorithm [23] the proposed algorithms provide better performance in 


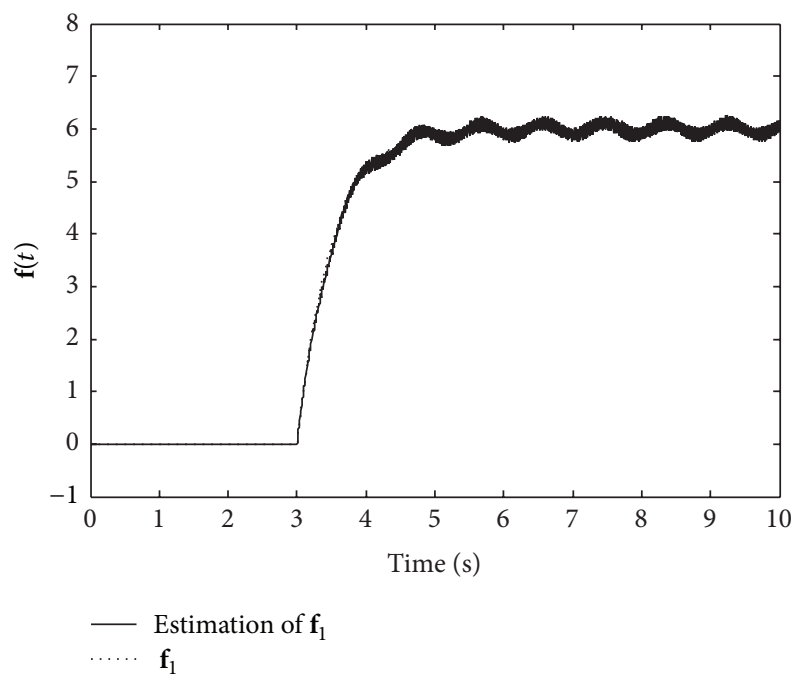

FIGURE 2: Single abrupt fault $\mathbf{f}_{1}(t)$ (dotted line) and its estimation $\widehat{\mathbf{f}}_{1}(t)$ (solid line) using conventional algorithm.

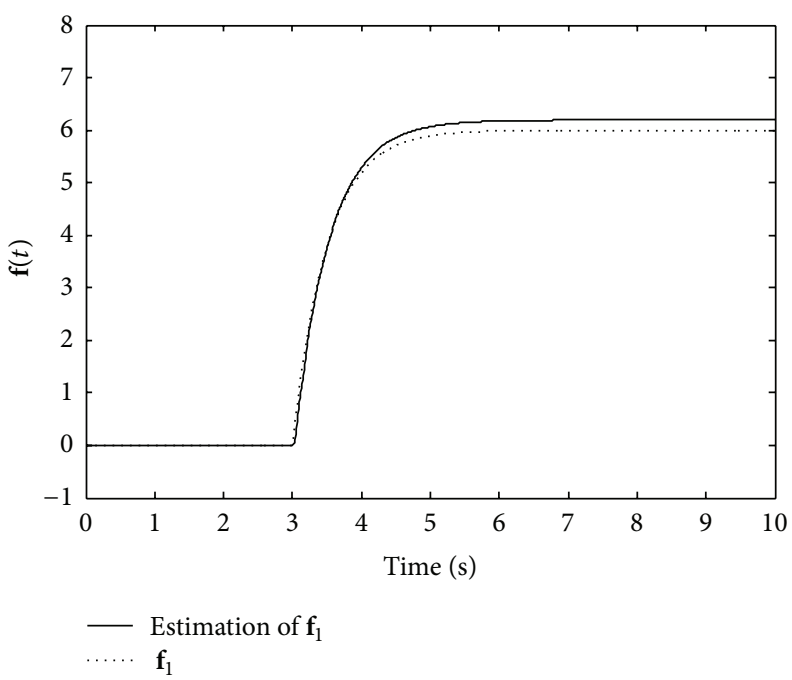

FIGURE 3: Single abrupt fault $\mathbf{f}_{1}(t)$ (dotted line) and its estimation $\widehat{\mathbf{f}}_{1}(t)$ (solid line) using AFEA.

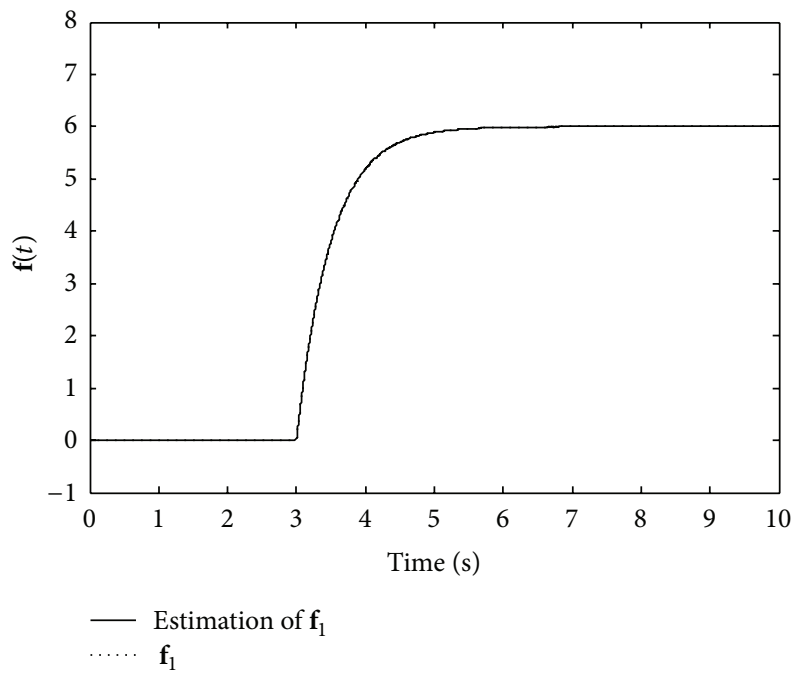

FIGURE 4: Single abrupt fault $\mathbf{f}_{1}(t)$ (dotted line) and its estimation $\widehat{\mathbf{f}}_{1}(t)$ (solid line) using improved AFEA. 


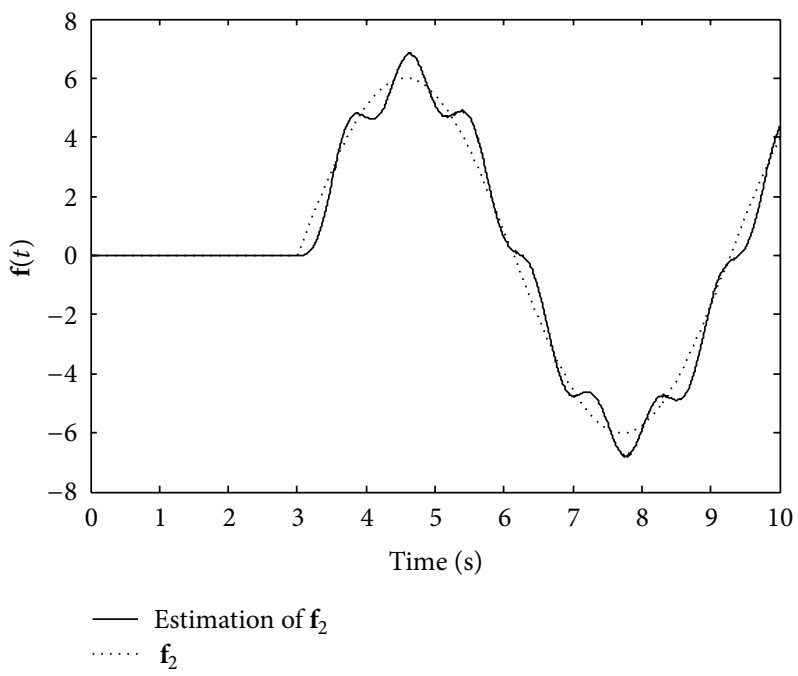

FIGURE 5: Single slow-varying fault $\mathbf{f}_{2}(t)$ (dotted line) and its estimation $\widehat{\mathbf{f}}_{2}(t)$ (solid line) using conventional algorithm.

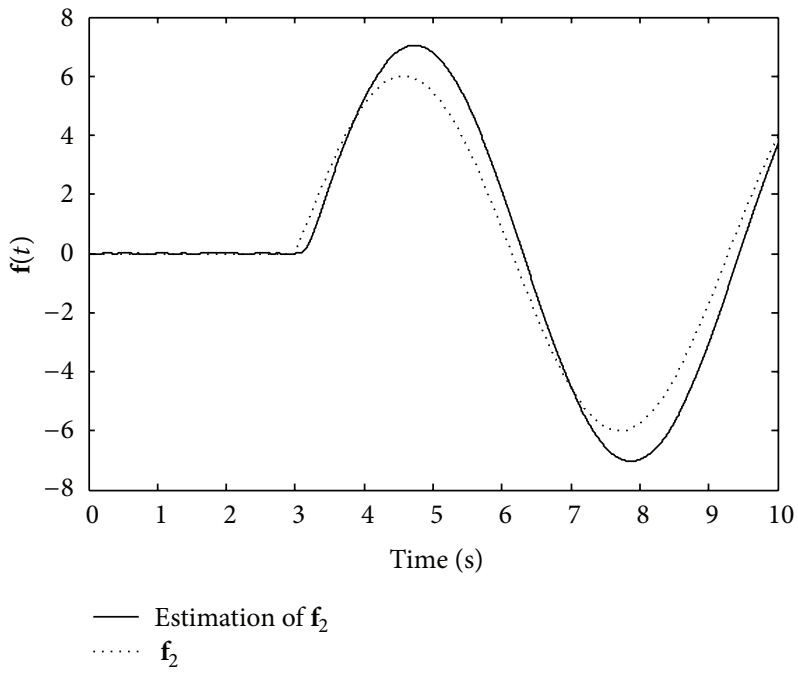

FIGURE 6: Single slow-varying fault $\mathbf{f}_{2}(t)$ (dotted line) and its estimation $\widehat{\mathbf{f}}_{2}(t)$ (solid line) using AFEA.

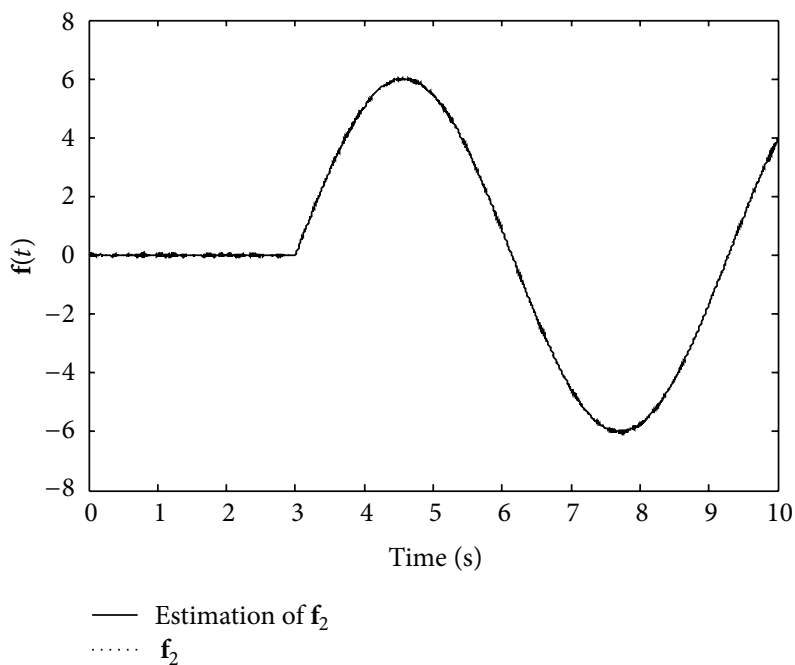

FIGURE 7: Single slow-varying fault $\mathbf{f}_{2}(t)$ (dotted line) and its estimation $\widehat{\mathbf{f}}_{2}(t)$ (solid line) using improved AFEA. 


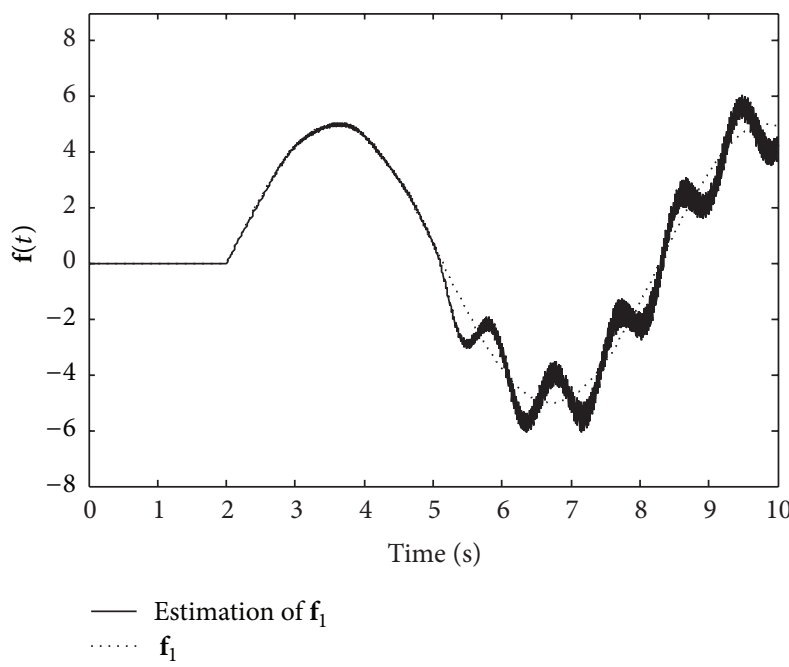

(a)

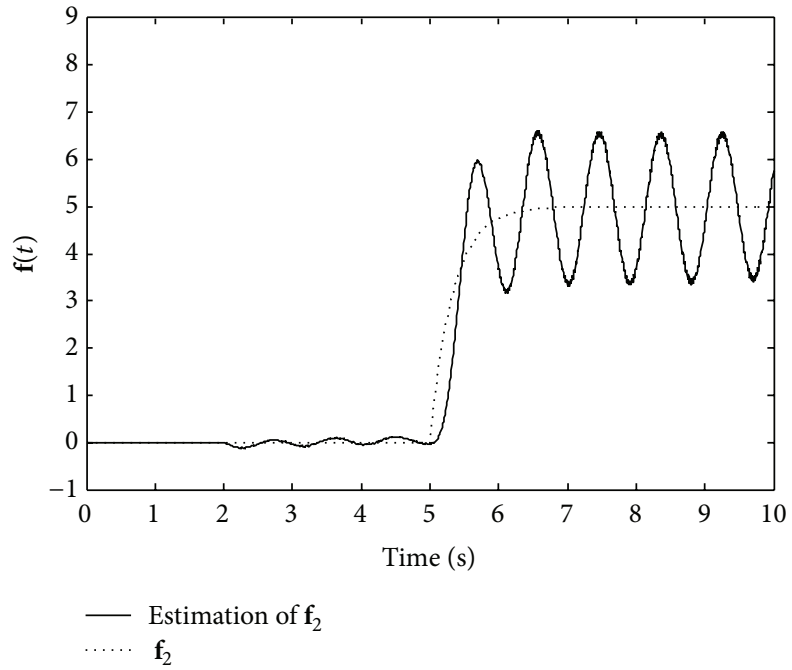

(b)

Figure 8: Combined fault $\mathbf{f}(t)$ (dotted line) and its estimation $\widehat{\mathbf{f}}(t)$ (solid line) using conventional algorithm.

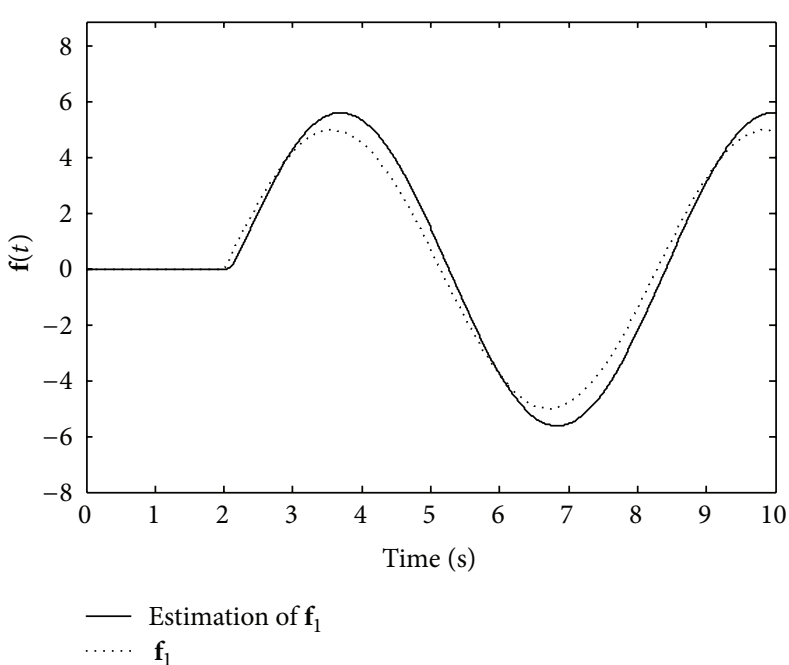

(a)

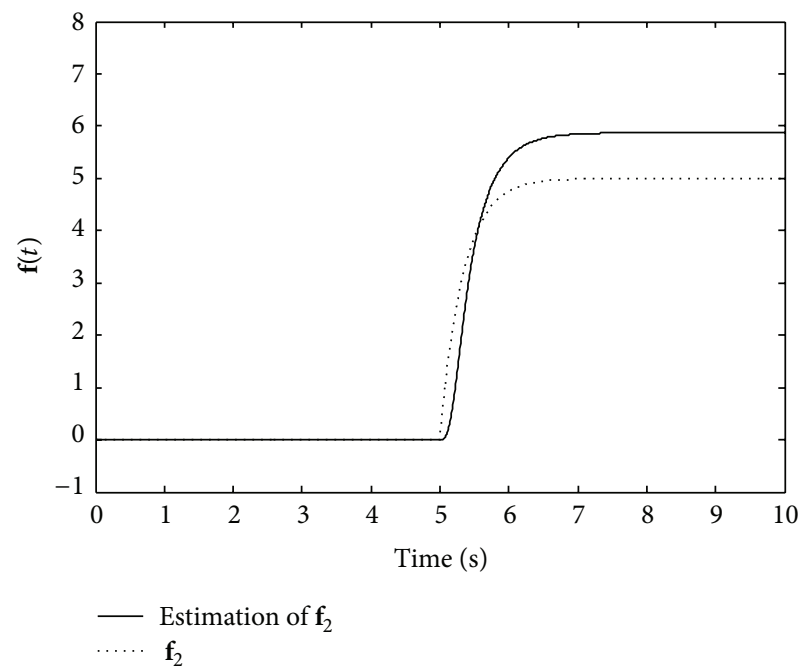

(b)

Figure 9: Combined fault $\mathbf{f}(t)$ (dotted line) and its estimation $\widehat{\mathbf{f}}(t)$ (solid line) using AFEA.

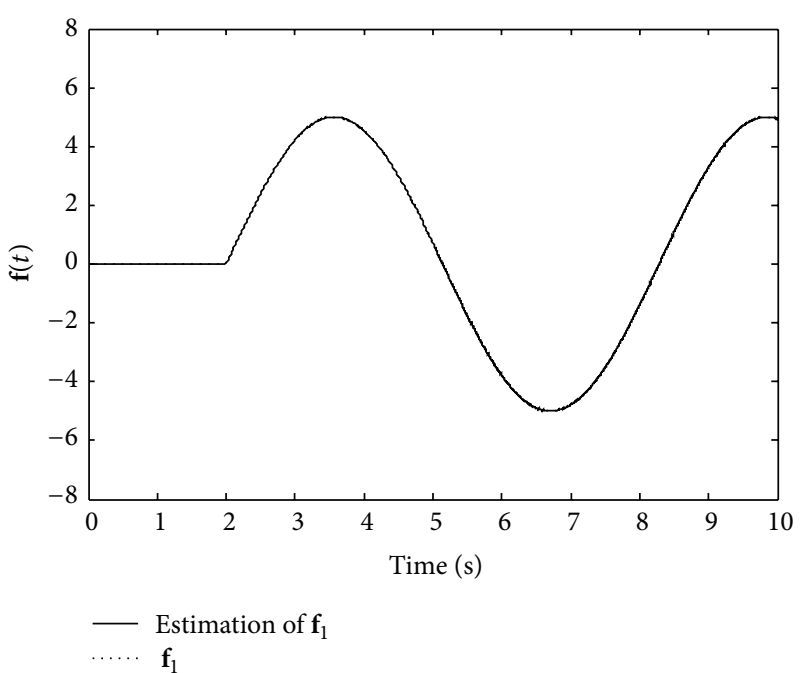

(a)

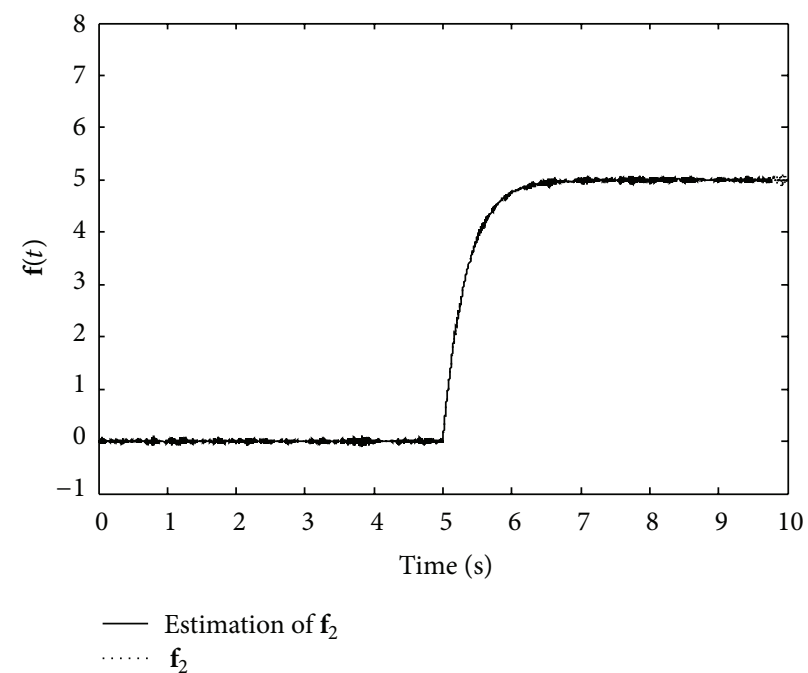

(b)

FIGURE 10: Combined fault $\mathbf{f}(t)$ (dotted line) and its estimation $\widehat{\mathbf{f}}(t)$ (solid line) using improved AFEA. 


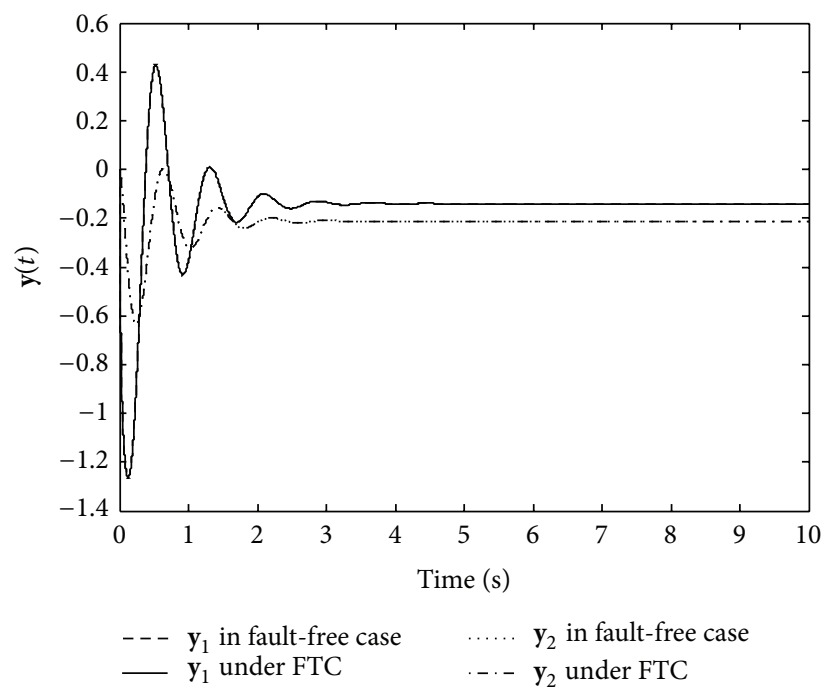

FIGURE 11: For single abrupt fault, system output response $\mathbf{y}(t)$.

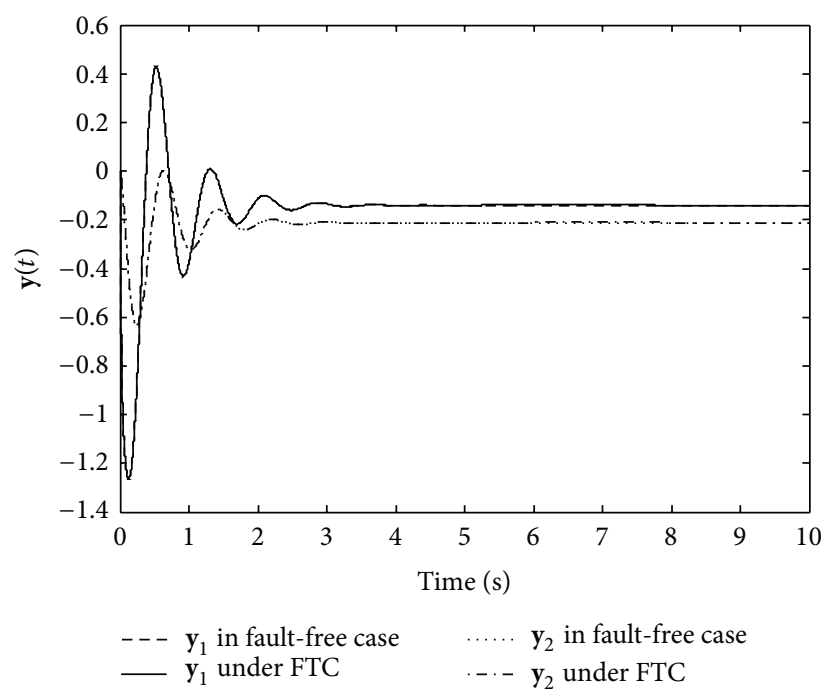

FIGURE 12: For single slow-varying fault, system output response $\mathbf{y}(t)$.

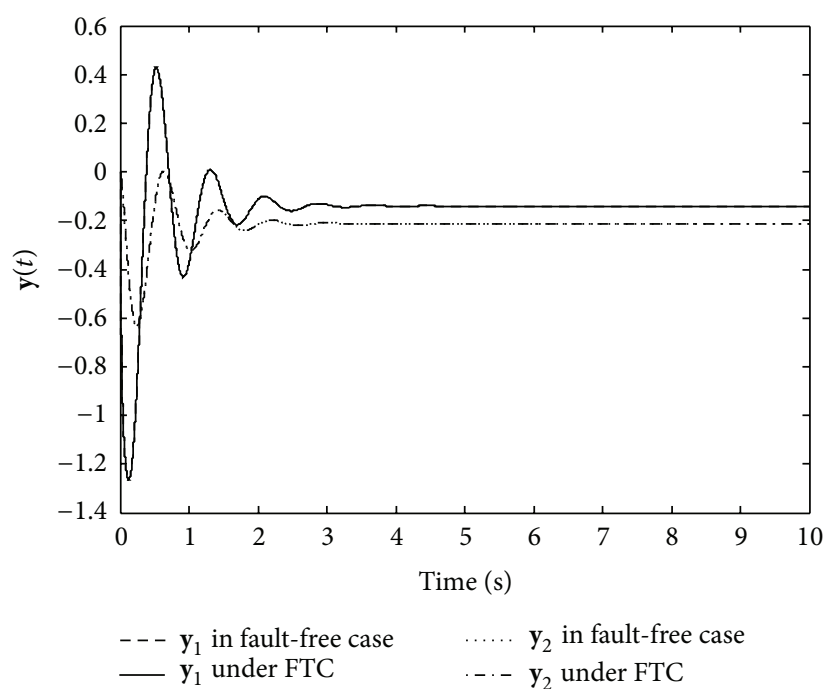

FIGURE 13: For combined fault, system output response $\mathbf{y}(t)$. 
fault estimation; particularly the improved AFEA enhances the rapidity and accuracy of fault estimation evidently. Then based on the IAFEO and compared with the system output responses in fault-free case, the designed OSFFTC can not only guarantee the stability of the closed-loop system, but also keep a desired performance in the presence of faults.

\section{Conclusion}

In this paper, a FTC strategy based on adaptive observer for a class of uncertain LTI systems has been proposed. An AFEA based on a normal fault estimator model is presented, which estimates states and faults by constructing an augmented observer. Then an improved AFEA utilizing a new fault estimator model is proposed, which uses LMI constraint technique to improve the rapidity and accuracy of state and fault estimation. By using the obtained on-line state and fault information, a state feedback fault-tolerant controller, which is independent of the IAFEO, is designed to stabilize the faulty system. Eliminating the coupling between the IAFEO and the fault-tolerant controller is meaningful and allows considering their properties separately. The application of this scheme to the lateral motion model of a light aircraft shows that actuator faults can be estimated with satisfactory performance and the fault-tolerant controller can guarantee the stability of the faulty system. Since most industrial systems are nonlinear, further research should focus more attention on extensions of the proposed approach, which can deal with more general nonlinear systems.

\section{Conflict of Interests}

The authors declare that there is no conflict of interests regarding the publication of this paper.

\section{Acknowledgments}

The authors would like to thank the editor and anonymous reviewers for their help, comments, and suggestions, which have helped to improve the presentation of this paper.

\section{References}

[1] Y. M. Zhang and J. Jiang, "Bibliographical review on reconfigurable fault-tolerant control systems," Annual Reviews in Control, vol. 32, no. 2, pp. 229-252, 2008.

[2] J. P. Ma and J. Jiang, "Applications of fault detection and diagnosis methods in nuclear power plants: a review," Progress in Nuclear Energy, vol. 53, no. 3, pp. 255-266, 2011.

[3] J. Jiang and X. Yu, "Fault-tolerant control systems: a comparative study between active and passive approaches," Annual Reviews in Control, vol. 36, no. 1, pp. 60-72, 2012.

[4] G.-H. Yang, J. L. Wang, and Y. C. Soh, "Reliable $H_{\infty}$ controller design for linear systems," Automatica, vol. 37, no. 5, pp. 717-725, 2001.

[5] C.-S. Hsieh, "Performance gain margins of the two-stage LQ reliable control," Automatica, vol. 38, no. 11, pp. 1985-1990, 2002.
[6] F. Liao, J. L. Wang, and G.-H. Yang, "Reliable robust flight tracking control: an LMI approach," IEEE Transactions on Control Systems Technology, vol. 10, no. 1, pp. 76-89, 2002.

[7] L.-L. Fan and Y.-D. Song, "On fault-tolerant control of dynamic systems with actuator failures and external disturbances," Acta Automatica Sinica, vol. 36, no. 11, pp. 1620-1625, 2010.

[8] G.-H. Yang and D. Ye, "Reliable $H_{\infty}$ control of linear systems with adaptive mechanism," IEEE Transactions on Automatic Control, vol. 55, no. 1, pp. 242-247, 2010.

[9] Y. M. Zhang and J. Jiang, "Active fault-tolerant control system against partial actuator failures," IEE Proceedings: Control Theory and Applications, vol. 149, no. 1, pp. 95-104, 2002.

[10] M. M. Kale and A. J. Chipperfield, "Stabilized MPC formulations for robust reconfigurable flight control," Control Engineering Practice, vol. 13, no. 6, pp. 771-788, 2005.

[11] J. Jiang and Y. Zhang, "Accepting performance degradation in fault-tolerant control system design," IEEE Transactions on Control Systems Technology, vol. 14, no. 2, pp. 284-292, 2006.

[12] H. Alwi and C. Edwards, "Fault tolerant control using sliding modes with on-line control allocation," Automatica, vol. 44, no. 7, pp. 1859-1866, 2008.

[13] H. Ltaief, E. Gabriel, and M. Garbey, "Fault tolerant algorithms for heat transfer problems," Journal of Parallel and Distributed Computing, vol. 68, no. 5, pp. 663-677, 2008.

[14] H. Alwi, C. Edwards, and C. P. Tan, "Sliding mode estimation schemes for incipient sensor faults," Automatica, vol. 45, no. 7, pp. 1679-1685, 2009.

[15] S. Varma and K. D. Kumar, "Fault tolerant satellite attitude control using solar radiation pressure based on nonlinear adaptive sliding mode," Acta Astronautica, vol. 66, no. 3-4, pp. 486-500, 2010.

[16] R. J. Patton, D. Putra, and S. Klinkhieo, "Friction compensation as a fault-tolerant control problem," International Journal of Systems Science, vol. 41, no. 8, pp. 987-1001, 2010.

[17] Q. Dong, M. Zhong, and S. X. Ding, "Active fault tolerant control for a class of linear time-delay systems in finite frequency domain," International Journal of Systems Science, vol. 43, no. 3, pp. 543-551, 2012.

[18] S. K. Spurgeon, "Sliding mode observers: a survey," International Journal of Systems Science, vol. 39, no. 8, pp. 751-764, 2008.

[19] X.-G. Yan and C. Edwards, "Robust decentralized actuator fault detection and estimation for large-scale systems using a sliding mode observer," International Journal of Control, vol. 81, no. 4, pp. 591-606, 2008.

[20] M. Saif, B. Ebrahimi, and M. Vali, "A second order sliding mode strategy for fault detection and fault-tolerant-control of a MEMS optical switch," Mechatronics, vol. 22, no. 6, pp. 696705, 2012.

[21] Z. Gao and D. W. C. Ho, "Proportional multiple-integral observer design for descriptor systems with measurement output disturbances," IEE Proceedings on Control Theory and Applications, vol. 151, no. 3, pp. 279-288, 2004.

[22] Z. Gao and S. X. Ding, "Fault estimation and fault-tolerant control for descriptor systems via proportional, multipleintegral and derivative observer design," IET Control Theory \& Applications, vol. 1, no. 5, pp. 1208-1218, 2007.

[23] B. Jiang, J. L. Wang, and Y. C. Soh, "An adaptive technique for robust diagnosis of faults with independent effects on system outputs," International Journal of Control, vol. 75, no. 11, pp. 792802, 2002. 
[24] B. Jiang, M. Staroswiecki, and V. Cocquempot, "Fault accommodation for nonlinear dynamic systems," IEEE Transactions on Automatic Control, vol. 51, no. 9, pp. 1578-1583, 2006.

[25] K. Zhang, B. Jiang, and V. Cocquempot, "Adaptive observerbased fast fault estimation," International Journal of Control, Automation and Systems, vol. 6, no. 3, pp. 320-326, 2008.

[26] K. Zhang, B. Jiang, and P. Shi, "Fast fault estimation and accommodation for dynamical systems," IET Control Theory \& Applications, vol. 3, no. 2, pp. 189-199, 2009.

[27] Z. H. Mao, B. Jiang, and P. Shi, "Fault-tolerant control for a class of nonlinear sampled-data systems via a euler approximate observer," Automatica, vol. 46, no. 11, pp. 1852-1859, 2010.

[28] K. Zhang, B. Jiang, and P. Shi, "Observer-based integrated robust fault estimation and accommodation design for discretetime systems," International Journal of Control, vol. 83, no. 6, pp. 1167-1181, 2010.

[29] X. D. Zhang, M. M. Polycarpou, and T. Parisini, "Fault diagnosis of a class of nonlinear uncertain systems with Lipschitz nonlinearities using adaptive estimation," Automatica, vol. 46, no. 2, pp. 290-299, 2010.

[30] K. Zhang, M. Staroswiecki, and B. Jiang, "Static output feedback based fault accommodation design for continuous-time dynamic systems," International Journal of Control, vol. 84, no. 2, pp. 412-423, 2011.

[31] B. Huo, S. Tong, and Y. Li, "Adaptive fuzzy fault-tolerant output feedback control of uncertain nonlinear systems with actuator faults," International Journal of Systems Science, vol. 44, no. 12, pp. 2365-2376, 2013.

[32] Q. Wu and M. Saif, "Robust fault detection and diagnosis in a class of nonlinear systems using a neural sliding mode observer," International Journal of Systems Science, vol. 38, no. 11, pp. 881-899, 2007.

[33] A. Fekih, H. Xu, and F. N. Chowdhury, "Neural networks based system identification techniques for model based fault detection of nonlinear systems," International Journal of Innovative Computing, Information and Control, vol. 3, no. 5, pp. 1073-1085, 2007.

[34] M. M. Polycarpou, "Fault accommodation of a class of multivariable nonlinear dynamical systems using a learning approach," IEEE Transactions on Automatic Control, vol. 46, no. 5, pp. 736-742, 2001.

[35] J. Chen and R. J. Patton, Robust Model-Based Fault Diagnosis for Dynamic Systems, Kluwer Academic Publishers, Norwell, Mass, USA, 1999.

[36] D. Z. Zheng, Linear System Theory, Tsinghua University Press, Beijing, China, 2nd edition, 2002.

[37] M. Chilali and P. Gahinet, " $H_{\infty}$ design with pole placement constraints: an LMI approach," IEEE Transactions on Automatic Control, vol. 41, no. 3, pp. 358-367, 1996.

[38] C. Edwards, X. G. Yan, and S. K. Spurgeon, "On the solvability of the constrained Lyapunov problem," IEEE Transactions on Automatic Control, vol. 52, no. 10, pp. 1982-1987, 2007. 


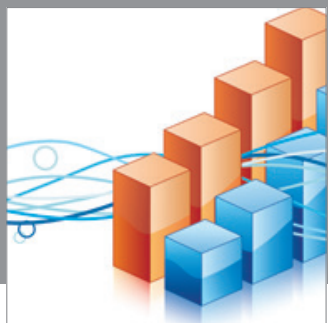

Advances in

Operations Research

mansans

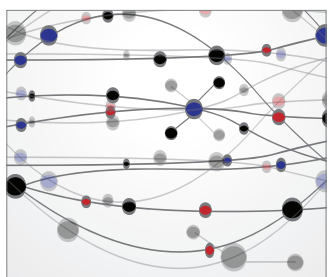

The Scientific World Journal
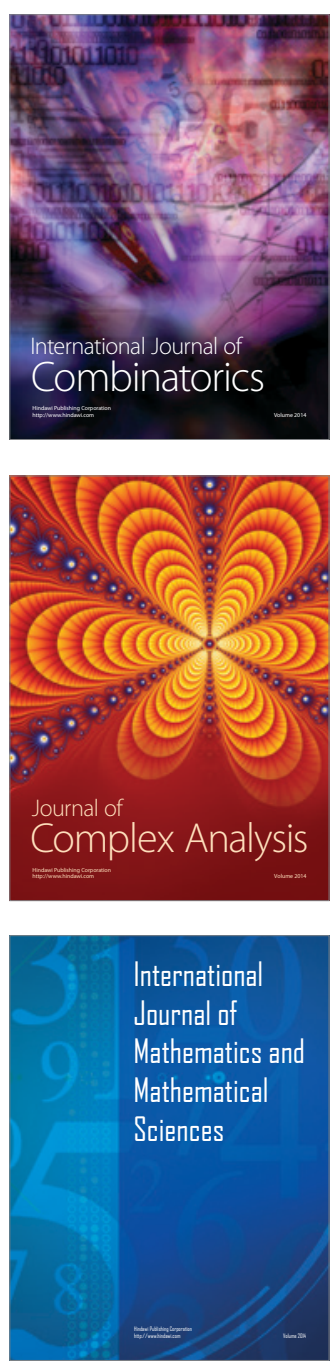
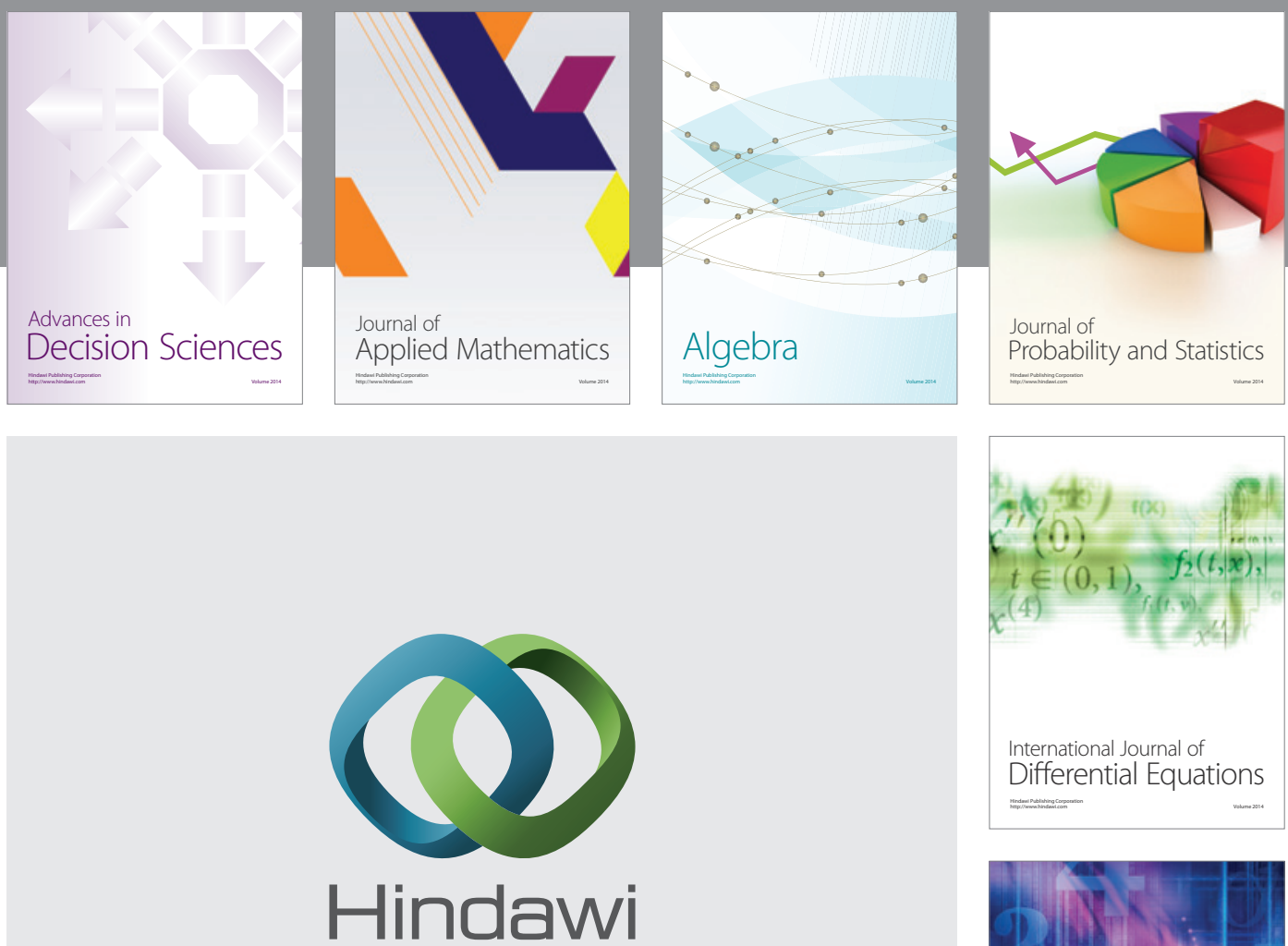

Submit your manuscripts at http://www.hindawi.com
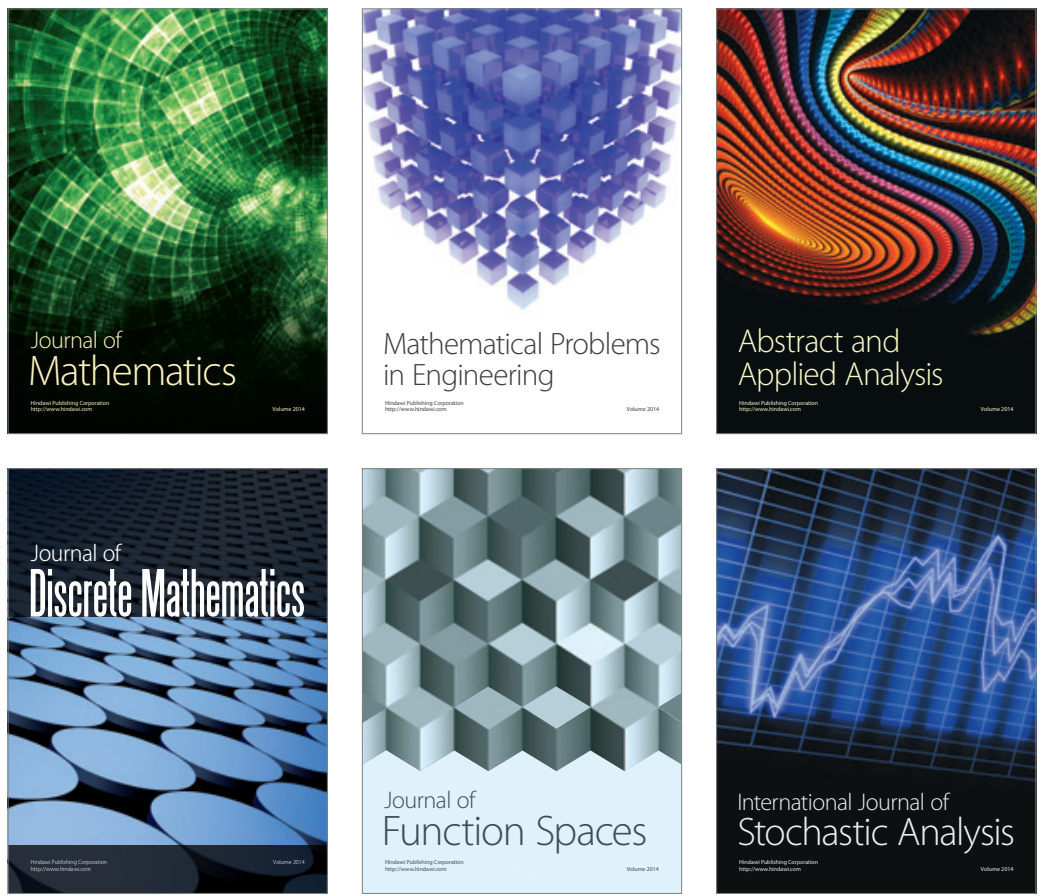

Journal of

Function Spaces

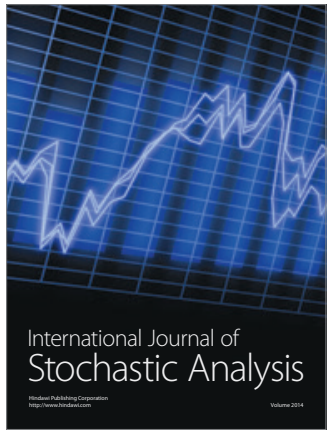

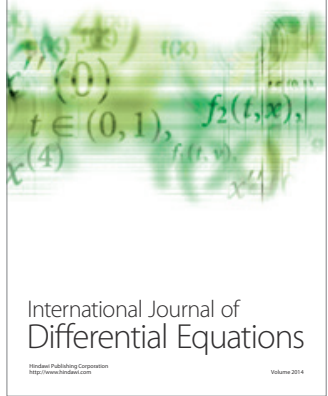
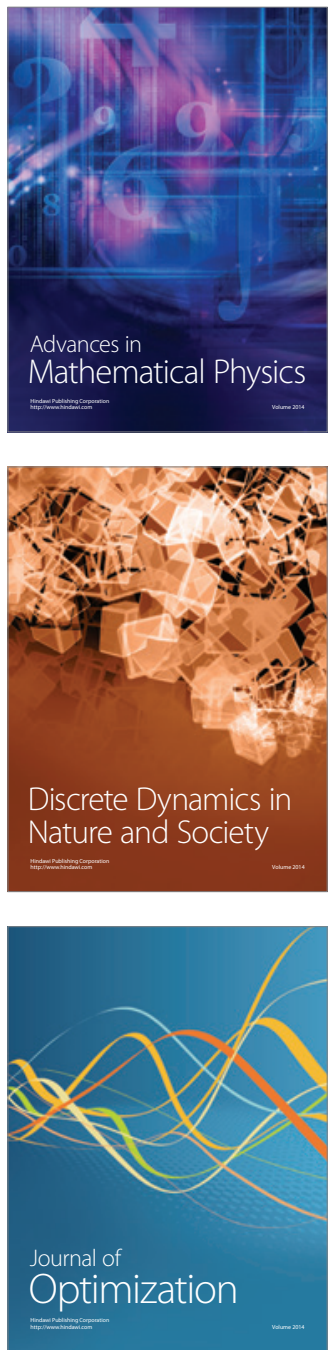